\title{
Oriented Bipartite Graphs and the Goldbach Graph
}

\author{
Sandip Das*, Prantar Ghosh , Shamik Ghosh $^{\ddagger}$ and Sagnik Sen ${ }^{\S}$
}

March 16, 2021

\begin{abstract}
In this paper, we study oriented bipartite graphs. In particular, we introduce "bitransitive" graphs. Several characterizations of bitransitive bitournaments are obtained. We show that bitransitive bitounaments are equivalent to acyclic bitournaments. As applications, we characterize acyclic bitournaments with Hamiltonian paths, determine number of non-isomorphic acyclic bitournaments of a given order, and solve the graph-isomorphism problem in linear time for acyclic bitournaments. Next, we prove the well-known Caccetta-Häggkvist Conjecture for oriented bipartite graphs for some cases for which it is unsolved in general oriented graphs. We also introduce the concept of undirected as well as oriented "odd-even" graphs. We characterize bipartite graphs and acyclic oriented bipartite graphs in terms of them. In fact, we show that any bipartite graph (acyclic oriented bipartite graph) can be represented by some odd-even graph (oriented odd-even graph). We obtain some conditions for connectedness of odd-even graphs. This study of odd-even graphs and their connectedness is motivated by a special family of odd-even graphs which we call "Goldbach graphs". We show that the famous Goldbach's conjecture is equivalent to the connectedness of Goldbach graphs. Several other number theoretic conjectures (e.g., the twin prime conjecture) are related to various parameters of Goldbach graphs, motivating us to study the nature of vertex-degrees and independent sets of these graphs. Finally, we observe Hamiltonian properties of some odd-even graphs related to Goldbach graphs for small number of vertices.
\end{abstract}

Keywords: Prime number; bipartite graph; directed bipartite graph; oriented bipartite graph; directed bitransitive graph; bitournament; Goldbach conjecture.

\section{Introduction}

A (simple) directed graph $D=(V, E)$ is bipartite if the vertex set $V$ is partitioned into $X$ and $Y$ such that there is no arc between any two vertices of $X$ or between any two vertices of $Y$. We

\footnotetext{
*Advanced Computing and Microelectronic Unit, Indian Statistical Institute, Kolkata, India. sandipdas@isical.ac.in

${ }^{\dagger}$ Department of Computer Science, Dartmouth College, Hanover, USA. prantar.ghosh.gr@dartmouth.edu

${ }^{\ddagger}$ Department of Mathematics, Jadavpur University, Kolkata, India. shamik.ghosh@jadavpuruniversity.in (Communicating author)

${ }^{\S}$ Department of Mathematics, Indian Institute of Technology Dharwad, Dharwad, India. sen@iitdh.ac.in
} 
usually denote such a graph by $D=(X, Y, E)$. A directed bipartite graph $D=(V, E)$ is oriented if for any $u, v \in V, u v \in E$ implies $v u \notin E$. An oriented bipartite graph $D=(X, Y, E)$ is called a bitournament if for all $x \in X$ and $y \in Y$, either $x y \in E$ or $y x \in E$. For a directed graph $D$, the undirected graph $G(D)$ obtained from $D$ by disregarding directions of arcs is the underlying graph of $D$. Moreover two arcs $e$ and $f$ of $D$ are adjacent if they have a common end point in $G(D)$. The adjacency matrix $M(D)$ of a directed bipartite graph, $D=(X, Y, E)$ is of the following form:

\begin{tabular}{|c|c|c|c|}
\hline \multirow{3}{*}{$M(D)=$} & & $X$ & $Y$ \\
\hline & $X$ & 0 & $A$ \\
\hline & $Y$ & $B$ & 0 \\
\hline
\end{tabular}

where $A$ and $B$ are two $(0,1)$-matrices. Note that in the case of an undirected bipartite graph, we have $B=A^{T}$, but it is not true in general for oriented bipartite graphs. $D$ is unidirectional if either $x y \notin E$ for all $x \in X, y \in Y$ or $y x \notin E$ for all $x \in X, y \in Y$. In this case either $A=\mathbf{0}$ or $B=\mathbf{0}$ in $M(D)$.

There are some interesting studies over directed bipartite graphs, oriented bipartite graphs, and in particular, oriented tress $[4,9,10,16]$. For a comprehesive study on bitournaments and in general, multipartite digraphs, one may consult the monograph [1], in particular, Chapter 2 [2] and Chapter 7 [18]. In this paper, we introduce bitransitive (directed) graphs. Several characterizations of bitransitive bitournaments are obtained. In particular, we show that bitounaments are bitransitive if and only if they are acyclic. As applications of the theorem, we characterize acyclic bitournaments with Hamiltonian paths, determine number of non-isomorphic acyclic bitournaments of a given order, and solve the isomorphism problem in linear time for acyclic bitournaments.

Next, we consider the Caccetta-Häggkvist Conjecture that states "Every simple directed graph of order $n$ with minimum outdegree at least $r$ has a cycle of length at most $\lceil n / r\rceil$." The conjecture is open for $r=n / 3, n / 4, n / 5$ and so on. We prove that the result is true for directed bipartite graphs for $r=n / 3, n / 4, n / 5$. Our main technical contribution is the $n / 5$ case, which requires some rigorous analysis of vertex-degrees.

We introduce the concept of oriented odd-even graphs and their undirected counterpart. We characterize the class of oriented bipartite graphs and (undirected) bipartite graphs in their terms. In fact, we show that any (acyclic oriented) bipartite graph can be represented by some (resp. oriented) odd-even graph. We obtain a necessary condition and another sufficient condition for connectedness of odd-even graphs. We study some cases where oriented odd-even graphs become unidirectional.

Finally, we introduce Goldbach graphs, a special family of odd-even graphs. We show that the famous Goldbach's conjecture is equivalent to the connectedness of Goldbach graphs. Furthermore, we observed that Maillet's, Kronecker's, and twin prime conjectures are related to various parame- 
ters of Goldbach graphs, especially to the vertex-degrees. So we study the nature of vertex-degrees and independent sets of Goldbach graphs. In the concluding section, we observe Hamiltonian properties of some odd-even graphs related to Goldbach graphs for small number of vertices and exhibit a sequence of even natural numbers up to 1000 such that for any pair of consecutive numbers in the sequence, one of them is the sum of two odd primes or 1 and the other is the difference between them (cf. Appendix B).

Throughout the paper let $\mathbb{N}$ denote the set of all natural numbers. If two natural numbers $a$ and $b$ are congruent modulo $p$, then we denote it by $a \equiv_{p} b$. Hence, in particular, for two numbers $a$ and $b$ with the same parity, we write $a \equiv_{2} b$, and if they have the opposite parity, we write $a \neq_{2} b$. We denote the set $\{1,2, \ldots, n\}$ by $[n]$ for any $n \in \mathbb{N}$.

\section{Oriented Bipartite Graphs}

In this section, we study several classes of oriented bipartite graphs. In Section 2.1, we introduce bitransitive digraphs and characterize bitransitive bitournaments. Next, in Section 2.2, we study acyclic bitournaments. Then, in Section 2.3, we prove the Caccetta-Häggkvist conjecture restricted to the class of oriented bipartite graphs for some cases that are open for general oriented graphs.

\subsection{Bitournaments and Bitransitive Digraphs}

We begin with an observation. Oriented trees form an interesting subclass of the class of oriented bipartite graphs. Let $T$ be an oriented tree. Then a path in the underlying tree $G(T)$ of $T$ is called alternating if each pair of adjacent arc are of opposite direction in $T$.

Observation 2.1. In an oriented tree $T$, there is an alternate path between any two vertices of $T$ if and only if for each vertex $v \in V(T)$, either indeg $(v)=0$ or outdeg $(v)=0$ (i.e., $T$ is unidirectional).

In the following, we introduce bitransitive bipartite digraphs, analogous to transitive general digraphs.

Definition 2.2 (Bitransitive Digraph). An oriented bipartite graph $D=(X, Y, E)$ is called bitransitive if for any $x_{1}, x_{2}, y_{1}, y_{2} \in X \cup Y, x_{1} y_{1}, y_{1} x_{2}, x_{2} y_{2} \in E \Longrightarrow x_{1} y_{2} \in E$ (see Figure 1 ).

We shall now define a digraph labelled by natural numbers that would work as an example of a bitransitive bitournament and shall help us to characterize them.

Definition 2.3 (Digraph $D_{S}$ ). Given a non-empty set $S \subseteq \mathbb{N}$, define $D_{S}$ as a digraph with the vertex set $S$ and the arc set $E=\left\{(a, b) \in S \times S \mid a<b\right.$ and $\left.a \not \equiv_{2} b\right\}$.

Example 2.4. For any non-empty set $S \subseteq \mathbb{N}, D_{S}=(X, Y, E)$ is a bitransitive bitournament with $X=\{u \in S \mid u$ is even $\}$ and $Y=\{u \in S \mid u$ is odd $\}$. 


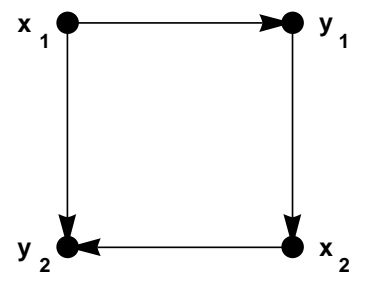

Figure 1: An illustration of bitransitive property

The following theorem characterizes bitransitive bitournaments. A Ferrers digraph $D=(V, E)$ is a directed graph whose successor sets are linearly ordered by inclusion where the successor set of $v \in V$ is its set of out-neighbors $\{u \in V \mid v u \in E\}$. It is known that a directed graph $D$ is a Ferrers digraph if and only if its adjacency matrix does not contain any $2 \times 2$ permutation matrix (called a couple) $[3,12]$ :

$$
\left[\begin{array}{ll}
1 & 0 \\
0 & 1
\end{array}\right] \quad \text { or } \quad\left[\begin{array}{ll}
0 & 1 \\
1 & 0
\end{array}\right] \text {. }
$$

Theorem 2.5. Let $D=(X, Y, E)$ be a bitournament. Then the following are equivalent:

1. $D$ is bitransitive.

2. D has no directed 4-cycle.

3. D has no directed cycle.

4. The matrix $M(D)$ is given by

\begin{tabular}{c|c|c|}
\multicolumn{1}{c}{} & \multicolumn{1}{c}{$X$} & $Y$ \\
\cline { 2 - 3 }$X$ & $\mathbf{0}$ & $A$ \\
\cline { 2 - 3 }$Y$ & $\bar{A}^{T}$ & $\mathbf{0}$ \\
\cline { 2 - 3 } & &
\end{tabular}

where $A$ is the adjacency matrix of a Ferrer's digraph and $\bar{A}$ is the 1 's complement of $A$.

5. $D \cong D_{S}$ (Definition 2.3) for some nonempty set $S \subseteq \mathbb{N}$.

Proof. $\mathbf{2} \Longrightarrow$ 1: Suppose there is no directed 4-cycle in a bitournament $D=(X, Y, E)$. Let $u_{1} u_{2}, u_{2} u_{3}, u_{3} u_{4} \in E$ for some $u_{1}, u_{2}, u_{3}, u_{4} \in V(D)=X \cup Y$. Then $u_{4} u_{1} \notin E$. Since $D$ is a bitournament, we have $u_{1} u_{4} \in E$. Hence it follows from Definition 2.2 that $D$ is bitransitive.

$1 \Longrightarrow$ 3: Suppose $D=(X, Y, E)$ is bitransitive but has a directed cycle. Since $D$ is bipartite, there cannot be any odd cycle. Hence the cycle is even. Now let the cycle be $\left(u_{1}, u_{2}, \ldots, u_{2 n}\right)$. We prove by induction that $u_{1} u_{2 k} \in E$ for all $k=1,2, \ldots, n$. By induction hypothesis, $u_{1} u_{2(k-1)} \in E$. Now $u_{2(k-1)} u_{2 k-1}, u_{2 k-1} u_{2 k}$. Hence $u_{1} u_{2 k} \in E$. So by induction, $u_{1} u_{2 k} \in E$ for all $k=1,2, \ldots, n$. 
Hence $u_{1} u_{2 n} \in E$. But we have already $u_{2 n} u_{1} \in E$. Since $D$ is a bitournament, both $u_{1} u_{2 n}, u_{2 n} u_{1}$ cannot be in $E$. Hence there is a contradiction.

$3 \Longrightarrow 2$ : Obvious.

$5 \Longrightarrow 2$ : Suppose $D \cong D_{S}$ for some nonempty set $S \subseteq \mathbb{N}$. Suppose it has a directed 4-cycle $\left(u_{1}, u_{2}, u_{3}, u_{4}\right)$. So $u_{1} u_{2}, u_{2} u_{3}, u_{3} u_{4}, u_{4} u_{1} \in E$. This implies $u_{1}<u_{2}<u_{3}<u_{4}<u_{1}$ which is a contradiction. So $D$ cannot have a directed 4 -cycle.

$\mathbf{2} \Longleftrightarrow \mathbf{4}$ : The adjacency matrix $A$ is not of a Ferrer's digraph if and only if there is a couple in $A$ such that

\begin{tabular}{l|c|c|}
\multicolumn{1}{c}{} & \multicolumn{1}{c}{$y_{r}$} & $y_{s}$ \\
\cline { 2 - 3 }$x_{i}$ & 1 & 0 \\
\cline { 2 - 3 }$x_{j}$ & 0 & 1 \\
\cline { 2 - 3 } & &
\end{tabular}

Hence $\bar{A}^{T}$ has the submatrix.

\begin{tabular}{lrl}
\multicolumn{1}{c}{} & \multicolumn{1}{c}{$x_{i}$} & \multicolumn{1}{c}{$x_{j}$} \\
\cline { 2 - 3 }$y_{r}$ & 0 & 1 \\
\cline { 2 - 3 }$y_{s}$ & 1 & 0 \\
\cline { 2 - 3 } & &
\end{tabular}

Thus, $x_{i} \rightarrow y_{r}, y_{r} \rightarrow x_{j}, x_{j} \rightarrow y_{s}$ and $y_{s} \rightarrow x_{i}$. Then we get a 4-cycle. Hence $A$ is not the adjacency matrix of a Ferrer's digraph if and only if there is a directed 4-cycle. That is, $A$ is the adjacency matrix of a Ferrer's digraph if and only if there is no directed 4-cycle.

$3 \Longrightarrow 5$ : We prove this by induction on number of vertices of a bitournament $D=(X, Y, E)$. The result is trivially true for 2 vertices, one in each partite set. Now suppose there are $n+1>2$ vertices in $D$. Now we remove a vertex $v$ from $D$. Then by induction hypothesis, the result is true for the resultant graph, say $D_{1}$ which has $n$ vertices, i.e., $D_{1} \cong D_{S}$ for some $\emptyset \neq S \subseteq \mathbb{N}$. Now, let $A$ be the set vertices $u$ of $D$ such that there is a directed path from $u$ to $v$. Let $B$ be the set of vertices $w$ of $D$ such that there is a directed path from $v$ to $w$. Since there is no directed cycle, $A$ and $B$ are disjoint. Now in $D_{S}$, any two vertices of opposite parity are adjacent so they are belonging to different partite sets in $D$. Thus $v$ cannot be adjacent to both of them. Let $v \in X$. Without loss of generality we may assume that other vertices of $X$ are labeled by even numbers in $D_{1}$ for otherwise we increase the label of each vertex in $D_{1}$ by 1 .

Let $m$ be an even number that is greater than all labels of vertices in $D_{1}$. We label $v$ as $m$ and for each $w \in B$, we relabel $w$ as $w+m$. We first note that adding $m$ does not change the parity for any $w$ in $B$. Next we prove that this relabeling does not violate the adjacency condition. Let there be an arc from $w \in B$ to a vertex $x$ in $D_{1}$. Then by construction $x \in B$. Hence all arcs from any $w \in B$ go to vertices to $B$ itself. Since the original labeling did not violate the adjacency condition, increasing each label by $m$ also does not violate it for arcs from some vertex of $B$ to another vertex of $B$. Now for the arcs from some $x \notin B$ to some $w \in B$, the adjacency condition is not violated 
as we have increased the label of $w$. All arcs from $v$ go to some vertex of $B$. Since $v=m$ and $w+m>m$, the adjacency condition is not violated for arcs from $v$ to some vertex of $B$. If there is an arc from a vertex $x$ to $v$, then $x \in A$ and since the label of $v$ is higher than any vertex of $A$, the adjacency retains. In all other cases, labels are not changed. Hence the relabeling matches the adjacency condition of any arc in $D$. This completes the proof.

The above characterization of acyclic bitournaments in terms of digraphs $D_{S}$ enables us to characterize acyclic bitournaments with Hamiltonian paths, determine number of non-isomorphic acyclic bitournaments of a given order, and solve the graph isomorphism problem for acyclic bitournaments in linear time. We show this in the following section.

\subsection{Acyclic Bitournaments}

In this section, we study the class of acyclic bitournaments (or, equivalently bitransitive bitournamemts). First, we show that an acyclic bitournament with a Hamiltonian path is unique (up to isomorphism) for a given order. Next, we show that the class of acyclic bitournaments can be given an "encoding" such that distinct (non-isomorphic) graphs from the class have distinct codes. This encoding enables us to count the number of non-isomorphic acyclic bitournaments and to check in linear time whether two given acyclic birtournaments are isomorphic.

Theorem 2.6. An acyclic bitournament $D$ with $n$ vertices has a Hamiltonian path if and only if $D$ is isomorphic to $D_{[n]}$, where $[n]:=\{1,2, \ldots, n\}$.

Proof. The "if" direction is immediate from the definition of $D_{S}$ (Definition 2.3). In $D_{[n]}$, we have the Hamiltonian path $1 \longrightarrow 2 \longrightarrow \cdots \longrightarrow n$.

For the "only if" direction, let $D$ be an acyclic bitournament which has a Hamiltonian path. By Theorem $2.5(\mathrm{v}), D \cong D_{S}$ for some nonempty set $S \subseteq \mathbb{N}$. Hence, $D_{S}$ has a Hamiltonian path, say $a_{1} \longrightarrow a_{2} \longrightarrow \ldots \longrightarrow a_{n}$. Now, in $D_{S}$, for every arc $x y$, we have $x<y$ and $x \not z_{2} y$. Thus, $a_{i}<a_{i+1}$ and $a_{i} \not \equiv_{2} a_{i+1}$ for each $i=1, \ldots, n-1$. Therefore, all elements in the set $\left\{a_{i} \mid i\right.$ is odd $\}$ have the same parity while all elements in the set $\left\{a_{i} \mid i\right.$ is even $\}$ have the opposite parity, i.e., $i \not \equiv_{2} j \Longleftrightarrow a_{i} \not \equiv_{2} a_{j}$. Let us map $i$ in $D_{[n]}$ to $a_{i}$ in $D_{S}$. We have

$$
i \rightarrow j \text { in } D_{[n]} \Longleftrightarrow i<j \text { and } i \neq_{2} j \Longleftrightarrow a_{i}<a_{j} \text { and } a_{i} \not \equiv_{2} a_{j} \Longleftrightarrow a_{i} \rightarrow a_{j} \text { in } D_{S}
$$

Hence, this is an isomorphism and $D_{S} \cong D_{[n]}$, i.e., $D \cong D_{[n]}$.

We now define a function that we shall use to encode acyclic bitournaments.

Definition 2.7 (Function $\beta_{S}$ ). Given a nonempty set $S \subseteq \mathbb{N}$, define the "scaling" function $\beta_{S}$ as follows. Let the increasing order of the natural numbers in $S$ be given by $\left\langle a_{1}, a_{2}, \ldots, a_{n}\right\rangle$. Then, $\beta_{S}: S \longrightarrow \mathbb{N}$ is defined inductively as $\beta_{S}\left(a_{1}\right)=1$, and for $i \geq 2, \beta_{S}\left(a_{i}\right)=\beta_{S}\left(a_{i-1}\right)+1$ if $a_{i} \not \equiv_{2} a_{i-1}$ and $\beta_{S}\left(a_{i}\right)=\beta_{S}\left(a_{i-1}\right)+2$ if $a_{i} \equiv_{2} a_{i-1}$. 
For a nonempty set $S \subseteq \mathbb{N}$ with $\left\langle a_{1}, a_{2}, \ldots, a_{n}\right\rangle$ being the increasing order of its elements, we define its "scaled" set $S_{\text {scl }}$ as

$$
S_{\mathrm{scl}}:=\left\{\beta_{S}\left(a_{1}\right), \beta_{S}\left(a_{2}\right), \ldots, \beta_{S}\left(a_{n}\right)\right\}
$$

Observation 2.8. $D_{S}$ is isomorphic to $D_{S_{s c l}}$.

Proof. For a digraph $D_{S}$, let the increasing order of the elements in $S$ be given by $\left\langle a_{1}, a_{2}, \ldots, a_{n}\right\rangle$ and that in $S_{\text {scl }}$ be given by $\left\langle b_{1}, b_{2}, \ldots, b_{n}\right\rangle$. Then, by definition of $S_{\text {scl }}$, for any $i, j \in[n]$, we have $b_{i}<b_{j}$ if and only if $a_{i}<a_{j}$ and $b_{i} \not \equiv_{2} b_{j}$ if and only if $a_{i} \not \equiv_{2} a_{j}$. Hence, by Definition 2.3, $D_{S} \cong D_{S_{\mathrm{scl}}}$.

The following lemma enables us to give a unique code to each acyclic bitournament.

Lemma 2.9. For two nonempty sets $S, T \subseteq \mathbb{N}, D_{S}$ is isomorphic to $D_{T}$ if and only if $S_{s c l}=T_{s c l}$.

Proof. One direction is obvious. $S_{\mathrm{scl}}=T_{\mathrm{scl}} \Longrightarrow D_{S_{\mathrm{scl}}} \cong D_{T_{\mathrm{scl}}} \Longrightarrow D_{S} \cong D_{T}$ (by Observation 2.8).

For the other direction, we proceed as follows. For a nonempty set $S$, denote the increasing order of its elements by $\left\langle a_{1}, \ldots, a_{n}\right\rangle$. Let $k$ be the integer such that $a_{1}, \ldots, a_{k}$ all have the same parity but $a_{k+1}$ has the opposite parity. Let $S^{\prime}:=S \backslash\left\{a_{1} \ldots, a_{k}\right\}$. We claim that $S_{\text {scl }}=\{1, \ldots, 2 k-1\} \cup$ $\left\{s^{\prime}+2 k-1: s^{\prime} \in S_{\mathrm{scl}}^{\prime}\right\}$.

First note that by definition, $\beta_{S}\left(a_{i}\right)=2 i-1$ for each $i \in[k]$. Let the increasing order of elements in $S_{\mathrm{scl}}^{\prime}$ be $\left\langle s_{1}^{\prime}, \ldots, s_{n-k}^{\prime}\right\rangle$. Now, we prove by induction that $\beta_{S}\left(a_{k+i}\right)=2 k-1+s_{i}^{\prime}$ for each $i \in[n-k]$. Since $a_{k+1} \not \equiv_{2} a_{k}$, we have $\beta_{S}\left(a_{k+1}\right)=\beta_{S}\left(a_{k}\right)+1=2 k-1+s_{1}^{\prime}$. Therefore, the base case holds. Now suppose $\beta_{S}\left(a_{k+j}\right)=2 k-1+s_{j}^{\prime}$ for some $j \geq 1$. Then, if $a_{k+j} \not_{2} a_{k+j+1}$, we have

$\beta_{S}\left(a_{k+j+1}\right)=\beta_{S}\left(a_{k+j}\right)+1=2 k-1+s_{j}^{\prime}+1=2 k-1+\beta_{S^{\prime}}\left(a_{k+j}\right)+1=2 k-1+\beta_{S^{\prime}}\left(a_{k+j+1}\right)=2 k-1+s_{j+1}^{\prime}$.

Again, if $a_{k+j} \equiv_{2} a_{k+j+1}$, we have

$\beta_{S}\left(a_{k+j+1}\right)=\beta_{S}\left(a_{k+j}\right)+2=2 k-1+s_{j}^{\prime}+2=2 k-1+\beta_{S^{\prime}}\left(a_{k+j}\right)+2=2 k-1+\beta_{S^{\prime}}\left(a_{k+j+1}\right)=2 k-1+s_{j+1}^{\prime}$.

Thus, we have proven the claim by induction. Next, suppose $D$ is isomorphic to $D_{S}$ and $D_{T}$ for two sets $S$ and $T$. Thus, $D_{S} \cong D_{T}$. Hence, $|S|$ must equal $|T|$. We prove that $S_{\text {scl }}=T_{\text {scl }}$ by induction on $n=|S|=|T|$. The base case holds for $n=2$, since there is only one nonempty bitournament on two vertices which is a single arc, and $S_{\mathrm{scl}}=T_{\mathrm{scl}}=\{1,2\}$. Now suppose the result is true for all $m \leq n-1$.

Since $D_{S}$ and $D_{T}$ are acyclic, they must have nonzero source vertices, i.e., vertices with in-degree 0 . Again, since they are isomorphic, they must have the same number (say $k$ ) of source vertices. Note that since these are source vertices, they must have the least values in $S$ and $T$ by Definition 2.3 and all of them must have the same parity. Let the digraphs obtained by deleting the source vertices from each of $D_{S}$ and $D_{T}$ be isomorphic to $D_{S^{\prime}}$ and $D_{T^{\prime}}$ respectively. Then since $D_{S} \cong D_{T}$, 
we have $D_{S^{\prime}} \cong D_{T^{\prime}}$. Therefore, since $\left|S^{\prime}\right|=\left|T^{\prime}\right|=n-k$, by the induction hypothesis, we have $S_{\mathrm{scl}}^{\prime}=T_{\mathrm{scl}}^{\prime}$. By the claim above, we have

$S_{\mathrm{scl}}=\{1, \ldots, 2 k-1\} \cup\left\{s^{\prime}+2 k-1: s^{\prime} \in S_{\mathrm{scl}}^{\prime}\right\}=\{1, \ldots, 2 k-1\} \cup\left\{t^{\prime}+2 k-1: t^{\prime} \in T_{\mathrm{scl}}^{\prime}\right\}=T_{\mathrm{scl}}$.

This completes the proof.

We are now ready to define the code of an acyclic bitournament.

Definition 2.10 (code $(D))$. Given an acyclic bitournament $D$, define code $(D)$ as the sequence obtained by taking the elements of $S_{\text {scl }}$ in increasing order, where $S$ is a set such that $D \cong D_{S}$.

Note that by Lemma 2.9, $\operatorname{code}(D)$ is a well-defined function. It then follows that code of an acyclic bitournament is unique up to isomorphism.

Lemma 2.11. Two acyclic bitournaments $D_{1}$ and $D_{2}$ are isomorphic if and only if code $\left(D_{1}\right)=$ $\operatorname{code}\left(D_{2}\right)$.

Proof. Let $D_{1}$ and $D_{2}$ be isomorphic to $D_{S}$ and $D_{T}$ for some nonempty sets $S, T \subseteq \mathbb{N}$ respectively. Then

$D_{1} \cong D_{2} \Longleftrightarrow D_{S} \cong D_{T} \Longleftrightarrow S_{\text {scl }}=T_{\text {scl }}($ by Lemma 2.9$) \Longleftrightarrow \operatorname{code}\left(D_{1}\right)=\operatorname{code}\left(D_{2}\right)($ by Definition 2.10).

Now, we shall use this encoding of the class of acyclic bitournaments to count of the number of non-isomorphic acyclic bitournaments of a given order.

Theorem 2.12. Let $\alpha$ be the number of non-isomorphic acyclic bitournaments $D=(X, Y, E)$. Then, $\alpha=\left(\begin{array}{c}2 n-1 \\ n\end{array}\right)$ when $|X|=|Y|=n$ and $\alpha=\left(\begin{array}{c}m+n \\ n\end{array}\right)$ when $|X|=m \neq n=|Y|$.

Proof. Consider the case that the acyclic bitournament is $D=(X, Y, E)$ with $|X|=|Y|=n$. By Lemma 2.11, we see that the number of non-isomorphic acyclic bitournaments is the number of distinct codes. Note that $\operatorname{code}(D)=\left\langle a_{1}, \ldots, a_{2 n}\right\rangle$ has $a_{1}=1$ and must contain $n$ even numbers and $n$ odd numbers as $|X|=|Y|=n$. For $i \in[n-1]$ let $k_{i}$ denote the number of even integers between the $i$ th and the $i+1$ th odd numbers in the sequence $\left\langle a_{1}, \ldots, a_{2 n}\right\rangle$, and $k_{n}$ be the number of even numbers after the $n$th odd number. We claim that two codes $\left\langle a_{1}, \ldots, a_{2 n}\right\rangle$ and $\left\langle a_{1}^{\prime}, \ldots, a_{2 n}^{\prime}\right\rangle$ differ if and only if their corresponding sequences $\left\langle k_{1}, \ldots, k_{n}\right\rangle$ and $\left\langle k_{1}^{\prime}, \ldots, k_{n}^{\prime}\right\rangle$ differ.

Let $a=\left\langle a_{1}, \ldots, a_{2 n}\right\rangle$ and $a^{\prime}=\left\langle a_{1}^{\prime}, \ldots, a_{2 n}^{\prime}\right\rangle$. If $a=a^{\prime}$, then clearly $\left\langle k_{1}, \ldots, k_{n}\right\rangle=\left\langle k_{1}^{\prime}, \ldots, k_{n}^{\prime}\right\rangle$. Now suppose that $\left\langle k_{1}, \ldots, k_{n}\right\rangle=\left\langle k_{1}^{\prime}, \ldots, k_{n}^{\prime}\right\rangle$. Then for each $i$, we prove that the two sets of numbers from the $i$ th to $i+1$ th odd numbers in the respective sequences $a$ and $a^{\prime}$ are equal. The base case for $i=0$ holds as $a_{1}=a_{1}^{\prime}=1$. Suppose it holds for some $i \geq 0$. Let the $i+1$ th odd number in $a$ and $a^{\prime}$ be $a_{j}$ and $a_{j}^{\prime}$ respectively. Now, we have $k_{i+1}=k_{i+1}^{\prime}$. If $k_{i+1}=k_{i+1}^{\prime}=0$, 
then, by definition, there is no even number between $a_{j}$ (equivalently $a_{j}^{\prime}$ ) and the next odd number in the sequence. Thus, $a_{j+1}$ (equivalently $\left.a_{j+1}^{\prime}\right)$ must be odd. Since $a_{j+1} \in\left\{a_{j}+1, a_{j}+2\right\}$ and $a_{j+1}^{\prime} \in\left\{a_{j}^{\prime}+1, a_{j}^{\prime}+2\right\}$, we must have $a_{j+1}=a_{j}+2=a_{j}^{\prime}+2=a_{j+1}^{\prime}$, where $a_{j+1}$ and $a_{j+1}^{\prime}$ are the $i+2$ th odd numbers in $a$ and $a^{\prime}$ respectively. Now consider the case when $k_{i+1}=k_{i+1}^{\prime}>0$. Then, there are $k_{i+1}$ even numbers in $a$ (resp. $a^{\prime}$ ) between $a_{j}$ (resp. $a_{j}^{\prime}$ ) and the next odd number. These numbers must be $a_{j}+1, a_{j}+3, \ldots, a_{j}+2 k_{i+1}-1$ and $a_{j}^{\prime}+1, a_{j}^{\prime}+3, \ldots, a_{j}^{\prime}+2 k_{i+1}-1$. The next odd number must then be $a_{j}+2 k_{i+1}$ and $a_{j}^{\prime}+2 k_{i+1}$ respectively. Since $a_{j}=a_{j}^{\prime}$, these two sets of numbers are equal. We have $a_{j+1}=a_{j+1}^{\prime}$. Hence, it follows by induction that for all $0 \leq i \leq n-1$, the numbers from the $i$ th to $i+1$ th odd numbers are equal in the sequences $a$ and $a^{\prime}$. Also since $\left\langle k_{1}, \ldots, k_{n}\right\rangle=\left\langle k_{1}^{\prime}, \ldots, k_{n}^{\prime}\right\rangle$, we have $a=a^{\prime}$.

Therefore, the number of distinct codes is the number of such sequences $\left\langle k_{1}, \ldots, k_{n}\right\rangle$. We see that the only constraints on $k_{i}$ are that they are non-negative and $\sum_{i=1}^{n} k_{i}=n$. Recall that the number of non-negative integer solutions to the equation $\sum_{i=1}^{r} x_{i}=s$ is $\left(\begin{array}{c}s+r-1 \\ s\end{array}\right)=\left(\begin{array}{c}s+r-1 \\ r-1\end{array}\right)$. Hence, there are $\left(\begin{array}{c}2 n-1 \\ n\end{array}\right)$ such sequences, i.e., $\left(\begin{array}{c}2 n-1 \\ n\end{array}\right)$ distinct codes, and hence there are $\left(\begin{array}{c}2 n-1 \\ n\end{array}\right)$ non-isomorphic acyclic bitournaments with partite sets of size $n$.

For the case when $|X|=m$ and $|Y|=n$ with $n \neq m, \operatorname{code}(D)=\left\langle a_{1}, \ldots, a_{n+m}\right\rangle$ has $a_{1}=1$ and either $n$ odd numbers and $m$ even numbers or vice versa. Then, by similar argument as above, the number of distinct codes is the number of sequences $\left\langle k_{1}, \ldots, k_{n}\right\rangle$ such that each $k_{i} \geq 0$ and $\sum_{i=1}^{n} k_{i}=m$ plus the number of sequences $\left\langle k_{1}^{\prime}, \ldots, k_{m}^{\prime}\right\rangle$ such that each $k_{i}^{\prime} \geq 0$ and $\sum_{i=1}^{m} k_{i}^{\prime}=n$. This is equal to $\left(\begin{array}{c}m+n-1 \\ n-1\end{array}\right)+\left(\begin{array}{c}m+n-1 \\ n\end{array}\right)=\left(\begin{array}{c}m+n \\ n\end{array}\right)$ (by Pascal's identity). Hence, there are $\left(\begin{array}{c}m+n \\ n\end{array}\right)$ non-isomorphic bitournaments in this case.

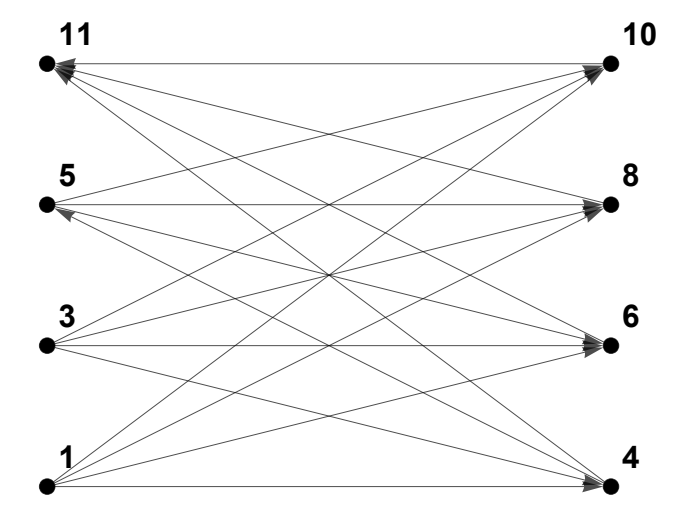

Figure 2: An acyclic bitournament with its code $\langle 1,3,4,5,6,8,10,11\rangle$.

Finally, we give a linear time algorithm to check isomorphism between two given acyclic bitourna- 
ments.

Theorem 2.13. There is a linear time algorithm for deciding whether two acyclic bitournaments are isomorphic.

Proof. Suppose we are given two acyclic bitournaments $D_{1}$ and $D_{2}$ as input and we need to check whether $D_{1} \cong D_{2}$. We describe an algorithm. For each of the digraphs, we do the following. Topologically sort it and obtain an ordering $\left\langle v_{1}, \ldots, v_{n}\right\rangle$ of the vertices. We set labels to the vertices using a function $\ell$ as follows. Set $\ell\left(v_{1}\right)=1$. For $i \geq 2$, if $v_{i-1}$ has an arc to $v_{i}$, then set $\ell\left(v_{i}\right)=\ell\left(v_{i-1}\right)+1$. Otherwise, set $\ell\left(v_{i}\right)=\ell\left(v_{i-1}\right)+2$. Call the sequences $\left\langle\ell\left(v_{1}\right), \ldots, \ell\left(v_{n}\right)\right\rangle$ obtained for $D_{1}$ and $D_{2}$ as $d_{1}$ and $d_{2}$ respectively. We decide that $D_{1} \cong D_{2}$ if and only if $d_{1}=d_{2}$.

We first argue the correctness. Let $S$ be the set $\left\{\ell\left(v_{1}\right), \ldots, \ell\left(v_{n}\right)\right\}$. Since $D$ is an oriented bipartite graph, we have assigned odd numbers to all vertices in the partite set containing $v_{1}$ and even numbers to all vertices in the other partite set. Thus for any arc $\left(v_{i}, v_{j}\right)$, we have $\ell\left(v_{i}\right) \not_{2} \ell\left(v_{j}\right)$. Again, because of the topological sorting, if $D$ has an $\operatorname{arc}\left(v_{i}, v_{j}\right)$, then $i<j$. Our construction ensures that if $i<j$, then $\ell\left(v_{i}\right)<\ell\left(v_{j}\right)$. Hence, for each arc $\left(v_{i}, v_{j}\right)$, we have $\ell\left(v_{i}\right)<\ell\left(v_{j}\right)$ and $\ell\left(v_{i}\right) \not \equiv_{2} \ell\left(v_{j}\right)$. Therefore, $D \cong D_{S}$. It follows from Definition 2.7 and the above construction that $S_{\text {scl }}=S$. Therefore, $d_{1}=\operatorname{code}\left(D_{1}\right)$. By similar argument, $d_{2}=\operatorname{code}\left(D_{2}\right)$. Thus, by Theorem 2.12 , we must have $D_{1} \cong D_{2}$ if and only if $d_{1}=d_{2}$.

Let us now analyze the runtime. Let $n=\left|V\left(D_{1}\right)\right|=\left|V\left(D_{2}\right)\right|$ and $m=\left|E\left(D_{1}\right)\right|=\left|E\left(D_{2}\right)\right|$. For digraphs $D_{1}$ and $D_{2}$, each topological sort takes $O(n+m)$. Constructing the labels for each digraph takes $O(n)$ time if the input is given in adjacency matrix form. If the input is of the form of adjacency list, then the construction of labels takes $\sum_{i=2}^{n}$ out-degree $\left(v_{i-1}\right)=O(m)$. Finally checking whether the codes are same takes $O(n)$ time. Hence, we get an $O(n+m)$ time, i.e., a linear time algorithm.

\subsection{Caccetta-Häggkvist Conjecture}

Here, we note that a conjecture for general directed graphs can be solved to some extent for directed bipartite graphs. The Caccetta-Häggkvist Conjecture states: "Every simple digraph of order $n$ with minimum outdegree at least $r$ has a cycle of length at most $\lceil n / r\rceil$." The conjecture has been proved for $r \leq \sqrt{n / 2}$ by Shen [13]. For $r \geq n / 2$ it is trivial since that means number of arcs in the graph is at least $n^{2} / 2>\left(\begin{array}{l}n \\ 2\end{array}\right)$, which implies the presence of a 2 -cycle. But it is still open for $r=n / 3, n / 4, n / 5$ and so on.

We consider the conjecture for directed bipartite graphs. For any $r<n$, if there exists a 2-cycle, we are done. So we can assume that the graphs are oriented bipartite graphs. Let $D=(X, Y, E)$ be an oriented bipartite graph with partite sets $X$ and $Y$, where $|X|=n_{1}$ and $|Y|=n_{2}\left(n_{1}, n_{2} \geq 1\right)$, 
and $E$ is the set of arcs. Let $V=X \cup Y$ be the set of vertices of $D$ with $|V|=n=n_{1}+n_{2}$. Consider the conjecture for $r=n / 3$. Since an oriented bipartite graph does not have a 3-cycle, the conjecture implies the following:

Proposition 2.14. There exists no oriented bipartite graph of order $n$ with minimum outdegree at least $n / 3$.

Proof. Suppose $d^{+}(v) \geq n / 3=\frac{n_{1}+n_{2}}{3} \forall v \in V$. Then $|E|=\sum_{v \in V} d^{+}(v) \geq \frac{\left(n_{1}+n_{2}\right)^{2}}{3} \geq \frac{4 n_{1} n_{2}}{3}>n_{1} n_{2}$ which is a contradiction since $|E| \leq n_{1} n_{2}$.

Now we have the following improvement of the above result.

Proposition 2.15. There exists no oriented bipartite graph of order $n$ with minimum outdegree $>n / 4$.

Proof. If $\forall v \in V, d^{+}(v) \geq n / 4$ and $\exists v_{0} \in V$ such that $d^{+}\left(v_{0}\right)>n / 4$, then $|E|=\sum_{v \in V} d^{+}(v)>$ $\frac{\left(n_{1}+n_{2}\right)^{2}}{4} \geq n_{1} n_{2}$ which is again a contradiction as before.

Thus, the above proposition can be restated as the following.

Corollary 2.16. In any oriented bipartite graph of order $n$, there exists a vertex with outdegree at most $n / 4$.

Now it follows that in an oriented bipartite graph with minimum outdegree $n / 4$, every vertex has outdegree exactly $n / 4$. Then, $n_{1} n_{2} \geq|E|=n^{2} / 4=\left(n_{1}+n_{2}\right)^{2} / 4 \geq n_{1} n_{2}$. Therefore, $|E|=n_{1} n_{2}=$ $\left(n_{1}+n_{2}\right)^{2} / 4$ and hence, $n_{1}=n_{2}$. Thus, we see that $D$ is an oriented complete bipartite graph, i.e., a bitournament with $|X|=|Y|$. Note that since $n / 4=n_{1} / 2$ is an integer (the exact outdegree of each vertex), $n_{1}$ must be even. Since the underlying undirected bipartite graph is complete, the in-degree of each vertex must also be $n_{1} / 2$. Therefore, for $r=n / 4$, the Caccetta-Häggkvist conjecture for oriented bipartite digraphs can be restated as the following.

Theorem 2.17. Let $D=(X, Y, E)$ be a bitournament with $|X|=|Y|=2 m$ and $d^{+}(v)=d^{-}(v)=$ $m \forall v \in V=X \cup Y$. Then D contains a 4-cycle.

Proof. Consider any 2-path $u \rightarrow v \rightarrow w$ in $D$ where $u, w \in X$ and $v \in Y$. Let $N(w) \subset Y$ be the set of $m$ out-neighbors of $w$. All vertices in $N(w)$ cannot be out-neighbors of $u$, otherwise $d^{+}(u) \geq|N(w) \cup\{v\}|=m+1$ which is a contradiction. Hence $\exists x \in N(w)$ such that $x$ is not an out-neighbor of $u$ and hence an in-neighbor of $u$. (Since every vertex in $Y$ is either an in-neighbor or an out-neighbor of $u$ ). Thus we have the 4-cycle $u \rightarrow v \rightarrow w \rightarrow x \rightarrow u$.

Remark 2.18. Note that in the case of Theorem 2.17, $D$ cannot be bitransitive by Theorem 2.5. 
We now prove the conjecture for the case $r=n / 5$. Since a bipartite graph cannot have a 5 -cycle, the case for $r=n / 5$ can be restated as: "An oriented bipartite graph $\left(X_{0}, X_{1}, E\right)$ with $\left|X_{0} \cup X_{1}\right|=n$ and minimum out-degree at least $n / 5$ has a directed 4-cycle."

We use some notations: for $i \geq 0$, let $N_{i}(v)$ denote the $i$ th neighborhood of a vertex $v$, i.e., the set of vertices which are at distance $i$ from $v$, and let $N_{-1}(v)$ denote the set of in-neighbors of the vertex $v$.

Lemma 2.19. In a bipartite graph with bipartition $\left(X_{0}, X_{1}\right)$ and minimum out-degree at least $n / 5$, if for some $i \in\{0,1\},\left|X_{i}\right| \leq \alpha_{1} n$ and $\left|X_{1-i}\right| \geq \alpha_{2} n$, then there exists $v \in X_{i}$ such that $\left|N_{-1}(v)\right| \geq \frac{\alpha_{2}}{5 \alpha_{1}} n$.

Proof. Since minimum out-degree of a vertex is at least $n / 5$, there are at least $\alpha_{2} n^{2} / 5$ outgoing arcs from $X_{1-i}$, which are "received" by at most $\alpha_{1} n$ vertices in $X_{i}$. Hence, by pigeon-hole principle, there exists a vertex $v \in X_{i}$ which "receives" at least $\frac{\alpha_{2} n^{2} / 5}{\alpha_{1} n}$ many arcs. Thus, $\left|N_{-1}(v)\right| \geq$ $\frac{\alpha_{2}}{5 \alpha_{1}} n$.

Lemma 2.20. Let $G=\left(X_{0}, X_{1}, E\right)$ be an oriented bipartite graph that does not contain a 4-cycle and has minimum out-degree at least $n / 5$. Let $v$ be a vertex in $X_{i}$, for some $i \in\{0,1\}$, such that $\left|N_{-1}(v)\right| \geq \alpha n$. Then

(i) $\left|N_{1}(v) \cup N_{3}(v)\right| \leq\left|X_{1-i}\right|-\alpha n$

(ii) $\left|N_{2}(v)\right| \geq \frac{0.04 n}{\frac{\left|X_{1-i}\right|}{n}-\alpha-0.2}$

Proof. Note that if $N_{3}(v) \cap N_{-1}(v) \neq \emptyset$, then there is a directed 4-cycle, which is a contradiction. Since the graph is oriented, we also have $N_{1}(v) \cap N_{-1}(v) \neq \emptyset$. Thus, $\left(N_{1}(v) \cup N_{3}(v)\right) \cap N_{-1}(v)=\emptyset$. Hence, $\left|N_{1}(v) \cup N_{3}(v)\right| \leq\left|X_{1-i}\right|-\left|N_{-1}(v)\right| \leq\left|X_{1-i}\right|-\alpha n$, which proves (i).

Now, consider the graph $G^{\prime}$ induced by $N_{2}(v) \cup\left(N_{1}(v) \cup N_{3}(v)\right)$. Since it is oriented, the number of arcs in $G^{\prime}$ is at most $\left|N_{2}(v)\right|\left|N_{1}(v) \cup N_{3}(v)\right| \leq\left|N_{2}(v)\right|\left(\left|X_{1-i}\right|-\alpha n\right)$. Again, the number of arcs in $G^{\prime}$ is at least the number of arcs "exiting" $N_{2}(v)$ and $N_{1}(v)$, which is at least $\left(\left|N_{1}(v)\right|+\left|N_{2}(v)\right|\right) \frac{n}{5} \geq$ $\left|N_{2}(v)\right| \frac{n}{5}+\frac{n^{2}}{25}$. Thus, we get the inequality $\left|N_{2}(v)\right| \frac{n}{5}+\frac{n^{2}}{25} \leq\left|N_{2}(v)\right|\left(\left|X_{1-i}\right|-\alpha n\right)$, which gives $\left|N_{2}(v)\right| \geq \frac{0.04 n}{\frac{\left|X_{1-i}\right|}{n}-\alpha-0.2}$, and this proves (ii).

Now we invoke Lemma 2.19 and Lemma 2.20 repeatedly to prove the following theorem:

Theorem 2.21. An oriented bipartite graph $G=\left(X_{0}, X_{1}, E\right)$ with $\left|X_{0} \cup X_{1}\right|=n$ and minimum out-degree at least $n / 5$ has a directed 4 -cycle.

Proof. Assume that $G$ does not contain a directed 4-cycle. WLOG, let $\left|X_{0}\right| \leq\left|X_{1}\right|$. We prove the theorem by considering the following cases: 
Case 1. $\left|X_{1}\right| \geq 0.75 n$.

Note that $\left|X_{0}\right| \leq 0.25 n$. By Lemma 2.19, $\exists v \in X_{0}$ such that $\left|N_{-1}(v)\right| \geq 0.6 n$. Now, $\left|N_{1}(v)\right| \geq n / 5$ and $N_{1}(v) \cap N_{-1}(v)=\emptyset$ since the graph is oriented. Again, $\left|N_{2}(v)\right| \geq n / 5$ and $v \notin N_{2}(v)$. Thus $|V| \geq\left|N_{-1}(v) \cup N_{1}(v) \cup N_{2}(v) \cup\{v\}\right| \geq 3 n / 5+n / 5+n / 5+1=n+1$, which is a contradiction.

Case 2. $0.65 n \leq\left|X_{1}\right|<0.75 n$.

Note that $0.25 n<\left|X_{0}\right| \leq 0.35 n$. By Lemma 2.19, $\exists v \in X_{0}$ such that $\left|N_{-1}(v)\right|>0.371 n$. Then, by Lemma 2.20, $\left|N_{1}(v) \cup N_{3}(v)\right|<0.379 n$ and $\left|N_{2}(v)\right|>0.223 n$. Again, by applying Lemma 2.19 on the induced bipartite graph with bipartition $\left(N_{1}(v) \cup N_{3}(v), N_{2}(v)\right), \exists u \in N_{1}(v) \cup N_{3}(v) \subset X_{1}$ such that $\left|N_{-1}(u)\right|>0.117 n$. Then, by Lemma $2.20,\left|N_{2}(u)\right|>\frac{0.04 n}{0.033}>n$, which is a contradiction.

Case 3. $0.6 n \leq\left|X_{1}\right|<0.65 n$.

Note that $0.35 n<\left|X_{0}\right| \leq 0.4 n$. By Lemma 2.19, $\exists v \in X_{0}$ such that $\left|N_{-1}(v)\right| \geq 0.3 n$. Then, by Lemma 2.20, $\left|N_{1}(v) \cup N_{3}(v)\right| \leq 0.35 n$ and $\left|N_{2}(v)\right|>0.26 n$. Again, by applying Lemma 2.19 on the induced bipartite graph with bipartition $\left(N_{1}(v) \cup N_{3}(v), N_{2}(v)\right), \exists u \in N_{1}(v) \cup N_{3}(v) \subset X_{1}$ such that $\left|N_{-1}(u)\right|>0.14 n$. Then, by Lemma $2.20,\left|N_{2}(u)\right|>0.66 \mathrm{n}$, which is a contradiction since $N_{2}(u) \subset X_{1}$ and $\left|X_{1}\right|<0.65 n$.

Case 4. $0.56 n \leq\left|X_{1}\right|<0.6 n$.

Note that $0.4 n<\left|X_{0}\right| \leq 0.44 n$. By Lemma 2.19, $\exists v \in X_{0}$ such that $\left|N_{-1}(v)\right|>0.254 n$. Then, by Lemma 2.20, $\left|N_{1}(v) \cup N_{3}(v)\right|<0.35 n$ and $\left|N_{2}(v)\right|>0.27 n$. Again, by applying Lemma 2.19 on the induced bipartite graph with bipartition $\left(N_{1}(v) \cup N_{3}(v), N_{2}(v)\right), \exists u \in N_{1}(v) \cup N_{3}(v) \subset X_{1}$ such that $\left|N_{-1}(u)\right|>0.154 n$. Then, by Lemma 2.20, $\left|N_{1}(v) \cup N_{3}(v)\right|<0.286 n$ and $\left|N_{2}(u)\right|>0.465 \mathrm{n}$. By applying Lemma 2.19 on the induced bipartite graph with bipartition $\left(N_{1}(u) \cup N_{3}(u), N_{2}(u)\right)$, $\exists w \in N_{1}(u) \cup N_{3}(u) \subset X_{0}$ such that $\left|N_{-1}(w)\right|>0.339 n$. Then, by Lemma $2.20,\left|N_{2}(w)\right|>0.655 \mathrm{n}$, which is a contradiction since $N_{2}(w) \subset X_{0}$ and $\left|X_{0}\right| \leq 0.44 n$.

Case 5. $0.53 n \leq\left|X_{1}\right|<0.56 n$.

Note that $0.44 n<\left|X_{0}\right| \leq 0.47 n$. By Lemma 2.19, $\exists v \in X_{0}$ such that $\left|N_{-1}(v)\right|>0.225 n$. Then, by Lemma 2.20, $\left|N_{1}(v) \cup N_{3}(v)\right|<0.335 n$ and $\left|N_{2}(v)\right|>0.296 n$. Again, by applying Lemma 2.19 on the induced bipartite graph with bipartition $\left(N_{1}(v) \cup N_{3}(v), N_{2}(v)\right), \exists u \in N_{1}(v) \cup N_{3}(v) \subset X_{1}$ such that $\left|N_{-1}(u)\right|>0.176 n$. Then, by Lemma 2.20, $\left|N_{1}(u) \cup N_{3}(u)\right|<0.294 n$ and $\left|N_{2}(u)\right|>0.425 n$. By applying Lemma 2.19 on the induced bipartite graph with bipartition $\left(N_{1}(u) \cup N_{3}(u), N_{2}(u)\right)$, $\exists w \in N_{1}(u) \cup N_{3}(u) \subset X_{0}$ such that $\left|N_{-1}(w)\right|>0.289 n$. Then, by Lemma $2.20,\left|N_{2}(w)\right|>0.563 n$, which is a contradiction since $N_{2}(w) \subset X_{0}$ and $\left|X_{0}\right| \leq 0.47 n$.

Case 6. $0.5 n \leq\left|X_{1}\right|<0.53 n$.

Note that $0.47 n<\left|X_{0}\right| \leq 0.5 n$. By Lemma 2.19, $\exists v \in X_{0}$ such that $\left|N_{-1}(v)\right| \geq 0.2 n$. Then, by Lemma 2.20, $\left|N_{1}(v) \cup N_{3}(v)\right|<0.33 n$ and $\left|N_{2}(v)\right|>0.307 n$. Again, by applying Lemma 2.19 on the induced bipartite graph with bipartition $\left(N_{1}(v) \cup N_{3}(v), N_{2}(v)\right), \exists u \in N_{1}(v) \cup N_{3}(v) \subset X_{1}$ such 
that $\left|N_{-1}(u)\right|>0.186 n$. Then, by Lemma 2.20, $\left|N_{1}(u) \cup N_{3}(u)\right|<0.314 n$ and $\left|N_{2}(u)\right|>0.35 n$. By applying Lemma 2.19 on the induced bipartite graph with bipartition $\left(N_{1}(u) \cup N_{3}(u), N_{2}(u)\right), \exists w \in$ $N_{1}(u) \cup N_{3}(u) \subset X_{0}$ such that $\left|N_{-1}(w)\right|>0.222 n$. Then, by Lemma 2.20, $\left|N_{1}(w) \cup N_{3}(w)\right|<0.308 n$ and $\left|N_{2}(w)\right|>0.37 n$. By applying Lemma 2.19 on the induced bipartite graph with bipartition $\left(N_{1}(w) \cup N_{3}(w), N_{2}(w)\right), \exists x \in N_{1}(w) \cup N_{3}(w) \subset X_{1}$ such that $\left|N_{-1}(x)\right|>0.24 n$. Then, by Lemma $2.20,\left|N_{2}(x)\right|>0.66 n$, which is a contradiction since $N_{2}(x) \subset X_{1}$ and $\left|X_{1}\right|<0.53 n$.

Hence, in each case we get a contradiction, but one of them must hold. Thus, our assumption that there is no 4-cycle in $G$ must be wrong. This completes the proof.

\section{Odd-even Graphs}

In this section, we introduce a family of graphs that we call odd-even graphs. Throughout the section we denote the set of all non-negative even numbers by $\mathcal{E}$ and the set of all positive odd numbers by $\mathcal{O}$. We begin with the definition of oriented odd-even graphs.

Definition 3.1. Let $A \subseteq \mathcal{E}$ and $O \subseteq \mathcal{O}$. An oriented odd-even graph $\overrightarrow{\mathcal{G}}_{A}(O)$ is an oriented graph with the set of vertices $A$ and with set of $\operatorname{arcs} E=\left\{a b \mid \frac{a+b}{2}, \frac{b-a}{2} \in O\right\}$.

Observe that $\overrightarrow{\mathcal{G}}_{A}(O)$ is an oriented bipartite graph with partite sets $V_{1}=\{v \in A \mid v \equiv 0(\bmod 4)\}$ and $V_{2}=\{v \in A \mid v \equiv 2(\bmod 4)\}$ as both $\frac{a+b}{2}$ and $\frac{b-a}{2}$ are even for any pair of $a, b \in V_{i}$ and for each $i \in\{1,2\}$.

Definition 3.2. An odd-even graph $\mathcal{G}_{A}(O)$ is the underlying (undirected) graph of $\overrightarrow{\mathcal{G}}_{A}(O)$, i.e., $\mathcal{G}_{A}(O)$ is a graph with set of vertices $A$ and with set of $\operatorname{arcs} E=\left\{a b \mid \frac{a+b}{2}, \frac{|a-b|}{2} \in O\right\}$.

From above, it is clear that $\mathcal{G}_{A}(O)$ is bipartite graph. Interestingly, the following theorem shows that every bipartite graph can be represented by an odd-even graph.

Theorem 3.3. Let $B$ be a bipartite graph. Then there exist $A \subseteq \mathcal{E}$ and $O \subseteq \mathcal{O}$ such that $\mathcal{G}_{A}(O)$ is isomorphic to $B$.

Proof. Let $B=(X, Y, E)$ be a bipartite graph with the partite sets $X$ and $Y$. Let $X=\left\{b_{0}, b_{2}, \ldots, b_{2 m}\right\}$, $Y=\left\{b_{1}, b_{3}, \ldots, b_{2 n-1}\right\}$ and $V=X \cup Y$. Now define a function $f: V \longrightarrow \mathcal{E}$ with $f\left(b_{i}\right)=$ $10^{i+2}+1+(-1)^{i+1}$. It is easy to check that the function $f$ is well-defined and injective. Take the even set $A$ to be the image of $f$ and let the odd set $O=\left\{\frac{f(a)+f(b)}{2}, \frac{|f(a)-f(b)|}{2} \mid a b \in E(B)\right\}$. Now to show that $B$ is isomorphic to $\mathcal{G}_{A}(O)$ it is enough to observe that $f(x)+f(y) \neq f\left(x^{\prime}\right)+f\left(y^{\prime}\right)$, $f(x)+f(y) \neq\left|f\left(x^{\prime}\right)-f\left(y^{\prime}\right)\right|,|f(x)-f(y)| \neq f\left(x^{\prime}\right)+f\left(y^{\prime}\right)$ and $|f(x)-f(y)| \neq\left|f\left(x^{\prime}\right)-f\left(y^{\prime}\right)\right|$ for any $x y \in E(B)$ and $x^{\prime} y^{\prime} \notin E(B)$.

Now from Definition 3.1 it is clear that $\overrightarrow{\mathcal{G}}_{A}(O)$ is acyclic, i.e., there is no directed cycle in $\overrightarrow{\mathcal{G}}_{A}(O)=$ $(V, E)$ as for any arc $a b \in E, b-a>0$ and so $a<b$. Thus no come back to the starting vertex 
is possible in a directed walk. Therefore $\overrightarrow{\mathcal{G}}_{A}(O)$ is an acyclic oriented bipartite graph. In the following we will see that any acyclic oriented bipartite graph can be represented by an oriented odd-even graph. Let $D=(V, E)$ be a digraph. An ordering $u_{1}, u_{2}, \ldots, u_{n}$ of vertices of $D$ is a topological ordering (or, acyclic ordering [6]) if for every arc $u_{i} u_{j} \in E$, we have $i<j$. It is known that every acyclic digraph has a topological ordering of vertices [6].

Theorem 3.4. Let $B$ be an acyclic oriented bipartite graph. Then there exist $A \subseteq \mathcal{E}$ and $O \subseteq \mathcal{O}$ such that $\overrightarrow{\mathcal{G}}_{A}(O)$ is isomorphic to $B$.

Proof. Let $B=(X, Y, E)$ be an acyclic oriented bipartite graph with the partite sets $X$ and $Y$ and $V=X \cup Y$. Let $u_{1}, u_{2}, \ldots, u_{n}$ be a topological ordering of $V$. Now we define a function $f: V \longrightarrow \mathcal{E}$ inductively. Assign $f\left(u_{1}\right)=10^{2}$ or $10^{3}+2$ according as $u_{1} \in X$ or $u_{1} \in Y$. Suppose $f\left(u_{i}\right)=10^{2 k}$ for some $k \in \mathbb{N}$. We assign $f\left(u_{i+1}\right)=10^{2 k+2}$ or $10^{2 k+1}+2$ according as $u_{i+1} \in X$ or $u_{i+1} \in Y$. If $f\left(u_{i}\right)=10^{2 k+1}+2$ for some $k \in \mathbb{N}$. We assign $f\left(u_{i+1}\right)=10^{2 k+2}$ or $10^{2 k+3}+2$ according as $u_{i+1} \in X$ or $u_{i+1} \in Y$. The function $f$ is well-defined and is a strictly increasing function (hence injective). We take the even set $A$ to be the image of $f$ and let the odd set $O=\left\{\frac{f(a)+f(b)}{2}, \frac{f(b)-f(a)}{2} \mid a b \in E(B)\right\}$. Since $f$ is increasing, $f(a)<f(b)$ for all $a b \in E$ due to the topological ordering. Then it follows that $\overrightarrow{\mathcal{G}}_{A}(O)$ is isomorphic to $B$ (rest of the proof is similar to the proof of Theorem 3.3).

Note that the above theorem can easily be extended to bipartite graphs with countably infinite number of vertices. Therefore, the family of odd-even graphs is, in fact, the family of all bipartite graphs with countable number of vertices. Now we will prove some conditions for finite odd even graphs to be connected. For any odd-even graph $\mathcal{G}_{A}(O)$, let the relevant odd set be $O_{\text {rel }}=O \cap$ $\left\{\frac{a+b}{2}, \frac{|a-b|}{2} \mid a b \in E\right\}$. Note that $\mathcal{G}_{A}(O)$ is isomorphic to $\mathcal{G}_{A}\left(O_{\text {rel }}\right)$.

Theorem 3.5. If $\mathcal{G}_{A}(O)$ is connected with $|A| \geq 2$, then $\left|O_{\text {rel }}\right| \geq \sqrt{2|A|}-1$.

Proof. Suppose $|A|=n$ and $\left|O_{\text {rel }}\right|=k$. Now, the number of edges in $\mathcal{G}_{A}(O)$ is at least $n-1$ (since $\mathcal{G}_{A}(O)$ is connected) and at most $\left(\begin{array}{l}k \\ 2\end{array}\right)+k=\frac{k(k+1)}{2}$. This is because each edge $a b$ corresponds to either a pair of numbers $\left(\frac{a+b}{2}, \frac{|a-b|}{2}\right) \in O_{\text {rel }}$ or a single number $\frac{a}{2} \in O_{\text {rel }}$ (in case $b=0$ ). Thus,

$$
\begin{array}{rlrl}
\frac{k(k+1)}{2} \geq n-1 & \Rightarrow\left(k-\frac{\sqrt{8 n-7}-1}{2}\right)\left(k+\frac{\sqrt{8 n-7}+1}{2}\right) \geq 0 & \\
& \Rightarrow k-\frac{\sqrt{8 n-7}-1}{2} \geq 0 & & \left(\text { since } k+\frac{\sqrt{8 n-7}+1}{2}>0\right) \\
& \Rightarrow k \geq \frac{\sqrt{8 n-7}-1}{2} \geq \sqrt{2 n}-1 \quad(\text { for } n \geq 2) \\
& \Rightarrow\left|O_{\text {rel }}\right| \geq \sqrt{2|A|}-1 .
\end{array}
$$


Theorem 3.6. Suppose $A=\{0,2,4, \ldots, 2(m-1)\}$. If $\left|O_{\text {rel }}\right| \geq \frac{7|A|}{8}$, then $\mathcal{G}_{A}(O)$ is connected.

Proof. WLOG, we can remove the isolated vertices from $A$ and prove the statement for the resulting set $A$. Since the size of $A$ can only decrease, the lower bound of $\frac{7|A|}{8}$ on $O_{r e l}$ still holds.

Assume to the contrary that $\mathcal{G}_{A}(O)$ is disconnected. Therefore, there exist at least two connected components. Let $X$ be a connected component and $Y$ be the union of the other connected components. Call the larger of these two sets as $Z$ and let $W$ be the other set. Then, we have $|Z| \geq \frac{|A|}{2}$. Now, $Z$ can be partitioned into two sets: those of the form $4 k+2$ and those of the form $4 k$. Let the larger set be $Z^{\prime}$. We must have $\left|Z^{\prime}\right| \geq \frac{|Z|}{2} \geq \frac{|A|}{4}$. Fix a vertex $a \in W$ such that $a$ has a form opposite to that of the numbers in $Z^{\prime}$. Note that such a number exists in $W$ because if $W$ has all numbers of the same form, then $W$ cannot have edges within itself, and hence, would be a set of isolated vertices. But we removed all such vertices.

For $b \in Z^{\prime}$, define $S_{b}=\left\{\frac{(a+b)}{2}, \frac{|a-b|}{2}\right\}$. Note that all elements in $S_{b}$ are odd. Define

$$
T=\left\{t \mid t \in S_{b} \text { for some } b \text { and } t \notin O_{r e l}\right\}
$$

We must have $O_{\text {rel }} \subseteq\{1,3, \ldots, 2 m-3\} \backslash T$, where $T \subseteq\{1,3, \ldots, 2 m-3\}$.

Observe that $a$ does not share edges with any vertex in $Z^{\prime}$. Therefore, at least one element from each $S_{b}$ does not belong to $O_{r e l}$. Consider the multiset obtained by adding the elements from each $S_{b}$ that do not belong to $O_{r e l}$. It has size at least $\left|Z^{\prime}\right| \geq \frac{|A|}{4}$, and the multiplicity of any number in this multiset can be at most 2 . Hence, removing duplicates, we get the set $T$, which has size at least $\frac{|A|}{8}$. Therefore, $\left|O_{\text {rel }}\right| \leq|\{1,3, \ldots, 2 m-3\}|-|T| \leq m-1-\frac{|A|}{8}=|A|-1-\frac{|A|}{8}<\frac{7|A|}{8}$. But this is a contradiction to our premise. Hence, $\mathcal{G}_{A}(O)$ must be connected.

Now we study odd-even graphs with odd sets of the following form:

$$
O_{a, b}=\{a k+b \mid a \in \mathcal{E}, b \in \mathcal{O}, k \in \mathbb{N}\} .
$$

Theorem 3.7. The oriented bipartite graph $\vec{G}=\overrightarrow{\mathcal{G}}_{\mathcal{E}}\left(O_{a, b}\right)=(V, E)$ is unidirectional if and only if 4 divides $a$.

Proof. Let $V_{1}=\{v \in \mathcal{E} \mid v \equiv 0(\bmod 4)\}$ and $V_{2}=\{v \in \mathcal{E} \mid v \equiv 2(\bmod 4)\}$. Then $V=V_{1} \cup V_{2}$. First assume that $a$ is divisible by 4 . Let $u=4 x \in V_{1}, v=4 y+2 \in V_{2}$ and $v u \in E$. So that forces $u>v$ as we have $\frac{u+v}{2}, \frac{u-v}{2} \in O_{a, b}$. That is, we have $2(x+y)+1,2(x-y)-1 \in O_{a, b}$. This implies

$$
2 x=a\left(n_{1}+n_{2}\right) / 2+b
$$

where $n_{1}, n_{2}$ are some positive integers. But this is a contradiction as $a\left(n_{1}+n_{2}\right) / 2+b$ is an odd number while $2 x$ is even. So all the arcs in $\vec{G}$ are from $V_{1}$ to $V_{2}$, i.e., $\vec{G}$ is unidirectional.

For the converse part, assume that $a$ is not divisible by 4 . Let $n_{1}>n_{2}$ be two positive even integers. Then $u=a\left(n_{1}-n_{2}\right) \in V_{1}$ and $v=a\left(n_{1}+n_{2}\right)+2 b \in V_{2}$. In this case, $\frac{u+v}{2}, \frac{v-u}{2} \in O_{a, b}$ and we 
have the arc $u v \in E$. On the other hand, consider two positive integers $m_{1}>m_{2}$ where $m_{1}$ is odd and $m_{2}$ is even. Then $u^{\prime}=a\left(m_{1}+m_{2}\right)+2 b \in V_{1}$ and $v^{\prime}=a\left(m_{1}-m_{2}\right) \in V_{2}$. In this case, $\frac{u^{\prime}+v^{\prime}}{2}, \frac{u^{\prime}-v^{\prime}}{2} \in O_{a, b}$ and we have the $\operatorname{arc} v^{\prime} u^{\prime} \in E$. So the graph $\vec{G}$ is not unidirectional when $a$ is not divisible by 4 .

Theorem 3.8. Let $\emptyset \neq I \subset \mathbb{N}$ and the odd set is given by $O=\left\{a_{i}+1 \mid a_{i} \in \mathcal{E}, i \in I\right\}$. Then the oriented graph $\overrightarrow{\mathcal{G}}_{\mathcal{E}}(O)$ is unidirectional if and only if 4 divides $a_{i}$ for all $i \in I$ or, 4 does not divide $a_{i}$ for all $i \in I$.

Proof. Let $V_{1}=\{v \in \mathcal{E} \mid v \equiv 0(\bmod 4)\}, V_{2}=\{v \in \mathcal{E} \mid v \equiv 2(\bmod 4)\}, V=V_{1} \cup V_{2}$ and $\overrightarrow{\mathcal{G}}_{\mathcal{E}}(O)=(V, E)$. First suppose that 4 divides $a_{i}$ for all $i \in I$. Let $u, v \in \mathcal{E}$ such that $u v \in E$. Then $\frac{u+v}{2}, \frac{v-u}{2} \in O$. Thus $\frac{u+v}{2}=4 k_{1}+1$ and $\frac{v-u}{2}=4 k_{2}+1$ for some $k_{1}, k_{2} \in \mathbb{N}$. These imply $v=4\left(k_{1}+k_{2}\right)+2$ and $u=4\left(k_{1}-k_{2}\right)$. Thus all the arcs are from $V_{1}$ to $V_{2}$.

Next consider that 4 does not divide $a_{i}$ for all $i \in I$. Let $u, v \in \mathcal{E}$ such that $u v \in E$. Then as before we have $\frac{u+v}{2}=4 k_{1}+3$ and $\frac{v-u}{2}=4 k_{2}+3$ for some $k_{1}, k_{2} \in \mathbb{N}$. These imply $v=4\left(k_{1}+k_{2}+1\right)+2$ and $u=4\left(k_{1}-k_{2}\right)$. Thus again we have all the arcs are from $V_{1}$ to $V_{2}$.

Finally let $a_{1}+1, a_{2}+1 \in O$ such that 4 divides $a_{1}$ and 4 does not divide $a_{2}$. Let $a_{1}>a_{2}$. Consider $u=a_{1}-a_{2}$ and $v=a_{1}+a_{2}+2$. Then $u \in V_{2}, v \in V_{1}, \frac{u+v}{2}=a_{1}+1$ and $\frac{v-u}{2}=a_{2}+1$. Thus $u v \in E$. Again for $w=2 a_{1}+2$, we have $0 w \in E$, where $0 \in V_{1}$ and $w \in V_{2}$. Thus the graph is not unidirectional. For $a_{1}<a_{2}$, the proof is similar with the choice $u=a_{2}-a_{1}$.

The adjacency matrix of the oriented graph $\overrightarrow{\mathcal{G}}_{\mathcal{E}}\left(O_{4,1}\right)$ is of the form

$$
\begin{array}{l|l}
\hline \mathbf{0} & X \\
\hline \mathbf{0} & \mathbf{0}
\end{array} \quad \text { where } \quad X=\left[\begin{array}{ccccccc}
1 & 0 & 1 & 0 & 1 & 0 & \ldots \\
0 & 1 & 0 & 1 & 0 & 1 & \ldots \\
0 & 0 & 1 & 0 & 1 & 0 & \ldots \\
0 & 0 & 0 & 1 & 0 & 1 & \ldots \\
0 & \ldots & & & & &
\end{array}\right]
$$

and the adjacency matrix of $\overrightarrow{\mathcal{G}}_{\mathcal{E}}\left(O_{6,1}\right)$ is

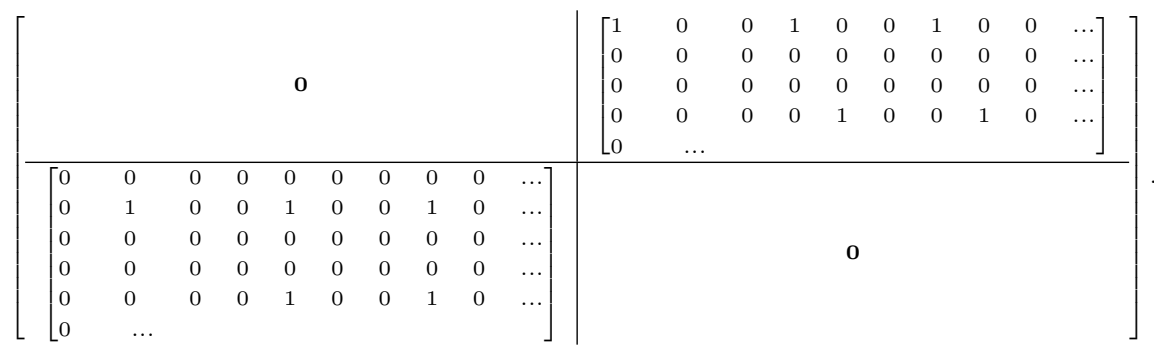

Note that according to Theorem 3.7, $\overrightarrow{\mathcal{G}}_{\mathcal{E}}\left(O_{4,1}\right)$ is unidirectional while $\overrightarrow{\mathcal{G}}_{\mathcal{E}}\left(O_{6,1}\right)$ is not. From the above two examples one can observe the difference between the adjacency matrices of unidirectional and not unidirectional oriented odd-even graphs. 


\section{The Goldbach Graph}

Here, we focus on a particular odd-even graph $\overrightarrow{\mathcal{G}}_{\mathcal{E}}(\mathcal{P})$ and $\mathcal{G}_{\mathcal{E}}(\mathcal{P})$ where the odd set $\mathcal{P}$ is the set of all odd primes, and call them the Goldbach (infinite) digraph and the Goldbach (infinite) graph respectively for the reason that will become apparent in the first result of this section. The set of vertices of the Goldbach (infinite) graph is the set of all non-negative even integers and two such vertices $a, b$ are adjacent if and only if both $\frac{a+b}{2}$ and $\frac{|a-b|}{2}$ are odd prime numbers. Let $\mathcal{E}_{n}$ denote the set of all non-negative even numbers less than or equal to $2 n$. Also, the graph $\mathcal{G}_{\mathcal{E}_{n}}(\mathcal{P})$ will be denoted by $\mathcal{G}_{n}$ and we call this graph a Goldbach (finite) graph. The neighborhood $N_{\mathcal{G}_{n}}(v$ ) (or, the out-neighbor $N_{\mathcal{G}_{n}}^{+}(v)$ or the in-neighbor $N_{\mathcal{G}_{n}}^{-}(v)$ ) of a vertex $v$ in $\mathcal{G}_{n}$ (or, in $\overrightarrow{\mathcal{G}}_{n}$ which we call a Goldbach (finite) digraph) will be denoted by $N_{n}(v)$ (or $N_{n}^{+}(v)$ or $N_{n}^{-}(v)$, respectively) for the remainder of the section. Also the degree $d_{\mathcal{G}_{n}}(v)$ (or, the out-degree $d_{\mathcal{G}_{n}}^{+}(v)$ or the in-degree $d_{\mathcal{G}_{n}}^{-}(v)$ ) of a vertex $v$ in $\mathcal{G}_{n}$ (or, in $\overrightarrow{\mathcal{G}}_{n}$ ) will be denoted by $d_{n}(v)$ (or $d_{n}^{+}(v)$ or $d_{n}^{-}(v)$, respectively) for the remainder of the section. We denote $\overrightarrow{\mathcal{G}}_{\mathcal{E}}(\mathcal{P})$ and $\mathcal{G}_{\mathcal{E}}(\mathcal{P})$ by $\overrightarrow{\mathcal{G}}_{\infty}$ and $\mathcal{G}_{\infty}$ respectively and the out-degree and the in-degree of $v \in \mathcal{E}$ in $\overrightarrow{\mathcal{G}}_{\infty}$ by $d_{\infty}^{+}(v)$ and $d_{\infty}^{-}(v)$ respectively and the degree of $v \in \mathcal{E}$ in $\mathcal{G}_{\infty}$ by $d_{\infty}(v) . N_{\infty}^{+}(v)$ or $N_{\infty}^{-}(v)$ are defined similarly. Now we state the result that, by and large, motivated this work.

Theorem 4.1. The following statements are equivalent.

(i) (Goldbach's conjecture) Every even integer greater than 5 can be written as sum of two odd primes.

(ii) $\mathcal{G}_{n}$ is connected for all $n \geq 7$.

(iii) $d_{\infty}^{-}(v)>0$ for all $v \geq 6$ in $\overrightarrow{\mathcal{G}}_{\infty}$.

Proof. (i) $\Rightarrow$ (ii): Suppose that the Goldbach's conjecture is true. Observe that $\mathcal{G}_{7}$ is connected. Now assume that $\mathcal{G}_{n}$ is connected for all $n \leq k$. By Goldbach Conjecture, $2(k+1)=p+q$ for some $p, q \in \mathcal{P}$. Then $|p-q|$ is even and $|p-q|<p+q=2(k+1)$. Thus $2(k+1)$ is adjacent to $|p-q|$ which is a vertex of $\mathcal{G}_{k}$ as well. This implies that $\mathcal{G}_{k+1}$ is connected.

(ii) $\Rightarrow$ (iii): Suppose $\mathcal{G}_{n}$ is connected for all $n \geq 7$. Let $v$ be any even integer greater equal to 14. Then, as the graph $\mathcal{G}_{v / 2}$ is connected, the vertex $v$ of the graph must be adjacent to some other vertex of the graph. Note that $v$ is the greatest vertex in $\mathcal{G}_{v / 2}$. Hence $d_{\infty}^{-}(v)>0$. Now it is a simple observation that for $6 \leq v \leq 12$ we have $d_{\infty}^{-}(v)>0$ as $0 \in N_{\infty}^{-}(6) \cap N_{\infty}^{-}(10)$ and $2 \in N_{\infty}^{-}(8) \cap N_{\infty}^{-}(12)$. This completes the proof.

(iii) $\Rightarrow(\mathbf{i})$ : Suppose $d_{\infty}^{-}(v)>0$ for all $v \geq 6$. Now for any even number $a>5$ there exists $b$ such that $b \in N_{\infty}^{-}(a)$. That means, there exist odd primes $p, q$ such that we have $p+q=a$. This is precisely the Goldbach's conjecture. 
The above result shows that the Goldbach's conjecture can be formulated using graph theoretic notions. Note that in Theorem 3.5 and 3.6 we presented one necessary and another sufficient conditions for connectedness of finite odd-even graphs. Improved results of similar nature might give rise to an alternative way of digging into the Goldbach's conjecture using graph theory due to Theorem 4.1. Having proved this equivalence, naturally we tried to explore more such equivalent formulations. Our observation which was integral in proving the above result is that, given a nonnegative even integer $2 n$, it is adjacent to a smaller integer implies that $2 n$ can be expressed as the sum of two odd primes. Similarly, its adjacency with a greater integer implies that $2 n$ can be expressed as difference of two odd primes. This readily provides graph theoretic formulation of another well-known conjecture in number theory.

Theorem 4.2. The following statements are equivalent.

(i) (A conjecture by Maillet [8]) Every non-negative even integer can be written as difference of two odd primes.

(ii) $d_{\infty}^{+}(v)>0$ for all $v \geq 2$ in $\overrightarrow{\mathcal{G}_{\infty}}$.

After this the first thing that came to our notice is that the degree of the vertices of our graph is particularly interesting. As the graph is an infinite graph, the natural question about the degrees are, if they are finite or not. In particular, note that each vertex have finite in-degree, as its inneighbors are smaller non-negative even numbers, while its out-degree can be unbounded. So the vertex 0 has no in-neighbors while its out-neighbors are precisely $2 p$ for all $p \in \mathcal{P}$. We know that there are infinitely many odd primes due to Euclid's theorem (which says, there are infinitely many prime numbers). Hence, $d_{\infty}^{+}(0)$ is infinite and this is equivalent to Euclid's theorem.

Observation 4.3. The vertex 0 of $\overrightarrow{\mathcal{G}}_{\infty}$ has infinitely many out-neighbors and hence, has infinitely many neighbors.

This observation naturally motivates us to wonder if the degree (or out-degree) of the other vertices are finite or not. It turns out to be a difficult question as it is equivalent to another well-known conjecture, the Kronecker's conjecture.

Theorem 4.4. The following statements are equivalent.

(i) (Kronecker's conjecture [7]) Given a positive even number $2 k$, there are infinitely many pairs of primes of the form $\{p, p+2 k\}$.

(ii) For every vertex $v \in \mathcal{E}$ we have $d_{\infty}^{+}(v)$ is infinite in $\overrightarrow{\mathcal{G}}_{\infty}$.

(iii) For every vertex $v \in \mathcal{E}$ we have $d_{\infty}(v)$ is infinite in $\mathcal{G}_{\infty}$. 
Proof. $(\mathbf{i}) \Rightarrow$ (ii): Suppose that the conjecture is true. Let $2 k$ be an even number for some $k \geq 1$. So, there are infinitely many pairs of primes of the form $\{p, p+2 k\}$ by assumption. Note that for each such pair of primes the vertex $2 k$ is adjacent to the vertex $2(p+k)$ in $\mathcal{G}_{\infty}$.

(ii) $\Leftrightarrow$ (iii): Clearly follows from the fact that $d_{\infty}^{+}(v) \leq d_{\infty}^{+}(v)+d_{\infty}^{-}(v)=d_{\infty}(v)$ for all $v \in \mathcal{E}$ while $d_{\infty}^{-}(v)$ is finite.

(iii) $\Rightarrow(\mathbf{i})$ : Suppose $d_{\infty}^{+}(v)$ is infinite for all $v \in \mathcal{E}$. Let $v=2 k$ be an even number for some $k \geq 1$. Now for each out-neighbor $u=2 n$ of $v$ in $\mathcal{G}_{\infty}$ we have $\frac{2 n+2 k}{2}, \frac{(2 n-2 k)}{2} \in \mathcal{P}$. Hence, both $(n-k)$ and $(n+k)$ are primes and there are infinitely such distincts pairs for each $k \geq 1$.

In particular, determining if degree (or out-degree) of 2 is finite or not will settle the twin prime conjecture [19] (positively if $d(2)$ is infinite). This implies an immediate corollary.

Corollary 4.5. The following statements are equivalent.

1. (Twin prime conjecture [19]) There are infinitely many pairs of primes of the form $\{p, p+2\}$.

2. $d_{\infty}^{+}(2)$ is infinite in $\overrightarrow{\mathcal{G}}_{\infty}$.

3. $d_{\infty}(2)$ is infinite in $\mathcal{G}_{\infty}$.

Next we will try to understand the significance of the degrees of the vertices in $\mathcal{G}_{\infty}$. Given a non-negative even number $2 n$, the in-degree $d_{\infty}^{-}(2 n)$ is the number of ways $2 n$ can be expressed as the sum of two odd primes. Similarly, the out-degree $d_{\infty}^{+}(2 n)$ is the number of ways $2 n$ can be expressed as the difference of two odd primes. Moreover, the degree of 0 in $\mathcal{G}_{n}$ is the number of odd primes less than or equal to $n$. So, the graph parameter $d_{n}(0)$ can be regarded as a function similar to the prime counting function $\pi(n)$, which denotes the number of primes less than or equal to $n$. So, for $n \geq 2$ we have

$$
\pi(n)=d_{n}(0)+1
$$

as the only even prime 2 is not adjacent to 0 . As it turned out to be an interesting yet difficult problem to figure out what the degrees of the vertices are, we started to establish some relations between them. Hence the following result.

Theorem 4.6. For all $n \geq 2 r$ and for $0 \leq m \leq 4$, in $\overrightarrow{\mathcal{G}}_{\infty}$ we have

$$
\sum_{i=0}^{m} d_{n}^{+}(2 i) \geq \sum_{i=0}^{m} d_{n}^{-}(2 r-2 i) .
$$

Sketch of the proof. Let $A_{i}=\{q \mid p+q=2 r-2 i$ and $q \leq p\}$ for $i \in\{0,1,2,3,4\}$ where $p, q$ are odd primes. Observe that $d_{n}^{-}(2 r-2 i)=\left|A_{i}\right|$. Note that for any $q \in \bigcup_{i=0}^{4} A_{i}$, we have $2 q \in N_{n}^{+}(0)$. Thus, $d_{n}^{+}(0) \geq\left|\bigcup_{i=0}^{4} A_{i}\right|$. 
Now suppose $q \in A_{i} \cap A_{j}$ for some $i, j \in\{0,1,2,3,4\}$ and $i<j$. Then there are primes $p_{1}, p_{2} \geq q$ such that $p_{1}+q=2 r-2 i$ and $p_{2}+q=2 r-2 j$. So $p_{2}=p_{1}-2(j-i)$. As both $\frac{2 p_{1}-2(j-i)+2(j-i)}{2}=p_{1}$ and $\frac{2 p_{1}-2(j-i)-2(j-i)}{2}=p_{1}-2(j-i)=p_{2}$ are odd primes we have

$$
\left.2 p_{1}-2(j-i)\right) \in N_{n}^{+}(2(j-i)) .
$$

Let $S_{i}=\left\{(i, x) \mid x \in N_{n}^{+}(2 i)\right\}$ for $i \in\{0,1,2,3,4\}$. Note that $S_{i} \cap S_{j}=\emptyset$ for $i \neq j$ and $\left|S_{i}\right|=d_{n}^{+}(2 i)$ for all $i \in\{0,1,2,3,4\}$. Also let $S=\bigcup_{i=0}^{4} S_{i}$. Then we will construct a subset $T \subseteq S$ such that $|T| \geq \sum_{i=0}^{4}\left|A_{i}\right|$. This will complete the proof.

Step 0: We know that for each $q \in \bigcup_{i=0}^{4} A_{i}$, we have $(0,2 q) \in S_{0}$. Put all these $(0,2 q)$ 's in the set $T$. Next we have to deal with the elements that are in more than one $A_{i}$ 's.

Step 1: First we handle the case where an element $q \in A_{0} \cap A_{j}$ for some $j \in\{1,2,3,4\}$. For each such $q$ there is a prime $p$ such that $p+q=2 r$. By (4.1) we know that that for each such $q$, there is an edge between $2 j$ and $(2 p-2 j)$. Put all these $(j, 2 p-2 j)$ 's in $T$. Observe that all these are new elements in $T$ as $j \geq 1$.

Step 2: Now consider an element $q \in A_{1} \cap A_{2}$. Then there exists a prime $p$ such that $p+q=2 r-2$ and by $(4.1)$ we know that $(1,2 p-2) \in S_{1}$. We will put all such $(1,2 p-2)$ 's in $T$ if they were already not in $T$. Let for some $q$, its corresponding $(1,2 p-2)$ were already in $T$. That means that element was included to $T$ due to Step 1 . Therefore, $p+(q+2)=2 r$ where $(q+2)$ is also a prime. Hence, $(1,2 q+2) \in S_{1}$ as both $\frac{2 q+2+2}{2}=(q+2)$ and $\frac{2 q+2-2}{2}=q$ are primes. Note that, all the elements included to $T$ before are of the form $(1,2 p-2)$ with $p \notin A_{0}$ while $(q+2) \in A_{0}$. Therefore, $(1,2 q+2)$ is not yet included to $T$. Now we include all such $(1,2 q+2)$ 's to $T$.

Step 3: Now consider an element $q \in A_{1} \cap A_{3}$. Then there exists a prime $p$ such that $p+q=2 r-2$ and by $(4.1)$ we know that $(2,2 p-4) \in S_{2}$. We will put all such $(2,2 p-4)$ 's in $T$ if they were not already in $T$.

Let for some $q$, its corresponding $(2,2 p-4)$ were already in $T$. That means that element was included to $T$ due to Step 1. An argument similar to Step 2 will show that there is an edge between $(2 q+2)$ and 2 . We will include all those $(1,2 q+2)$ 's to $T$ which were not included to $T$ before.

There may be some $(1,2 q+2)$ which was included to $T$ before. Then that inclusion was due to Step 2. This implies $p,(p-2)$ and $(p-4)$ are all odd primes. The only such instance is when $p=7$. Thus, $2 r-6=(p-4)+q=3+q$. As $(p-4) \geq q$ we have $q=3$. Hence, $2 r=12$. It is easy to check that the theorem holds for $2 r=12$. Therefore, we can ignore this case.

There are four more steps, namely, for $q \in A_{1} \cap A_{4}, q \in A_{2} \cap A_{3}, q \in A_{2} \cap A_{4}$ and $q \in A_{3} \cap A_{4}$ in that order, that will conclude the proof. Those cases can be handled in a similar way like above.

Our interest in the degree of the bipartite graph $\mathcal{G}_{\infty}$ prompted us to study the complete bipartite subgraphs of $\mathcal{G}_{\infty}$ from number theoretic point of view.

Proposition 4.7. If the complete bipartite graph $K_{m, n}$ is a subgraph of $\mathcal{G}_{\infty}$, then there exists a set $\left\{p_{1}, p_{2}, \ldots, p_{m}\right\}$ of $m$ primes and a set $\left\{r_{1}, r_{2}, \ldots r_{n-1}\right\}$ of $(n-1)$ positive integers such that $p_{i}+r_{j}$ 
is a prime for all $(i, j) \in\{1,2, \ldots, m\} \times\{1,2, \ldots, n\}$.

Proof. Let $X$ and $Y$ be the two partite sets of $K_{m, n}$. Index the vertices of $X=\left\{x_{1}, x_{2}, \ldots, x_{m}\right\}$ and $Y=\left\{y_{1}, y_{2}, \ldots, y_{n}\right\}$ in increasing order. Let $p_{i}=\frac{x_{i}+y_{1}}{2}$ and $r_{j}=\frac{y_{j+1}-y_{1}}{2}$ for $(i, j) \in\{1,2, \ldots, m\} \times$ $\{1,2, \ldots, n-1\}$. Note that $p_{i}+r_{j}=\frac{x_{i}+y_{j+1}}{2}$ is a prime for each $(i, j) \in\{1,2, \ldots, m\} \times\{1,2, \ldots, n-$ $1\}$.

Now we will prove some conditions for a complete bipartite subgraph of $\mathcal{G}_{\infty}$ with the aid of the following two lemmas. Let us denote $\mathbb{N} \cup\{0\}$ by $\mathbb{N}_{0}$.

Lemma 4.8. Let $a, b \notin 6 \mathbb{N}_{0}$ and $a b \in E\left(\mathcal{G}_{\infty}\right)$, then $|a-b|=6$.

Proof. Let $a>b$. Then $a=p+q$ and $b=p-q$ for some odd primes $p$ and $q$. If $p, q \neq 3$, then $p$ and $q$ are each of the form $6 k+1$ or $6 k-1$. Then either $6 \mid(p+q)=a$ or $6 \mid(p-q)=b$ contradicting the assumption of the lemma. Hence $q=3$ (as $p \geq q \geq 3$ ) which implies $a-b=2 q=6$.

Lemma 4.9. Let $a, b \in 6 \mathbb{N}_{0}$ and $a b \in E\left(\mathcal{G}_{\infty}\right)$ with $a \geq b$, then $a=6$ and $b=0$.

Proof. As both $a$ and $b$ are divisible by 6 , both $\frac{a+b}{2}$ and $\frac{a-b}{2}$ are divisible by 3 . Since they are primes, $\frac{a+b}{2}=3=\frac{a-b}{2}$. Therefore, $a=6, b=0$.

Theorem 4.10. Let $K_{m, n}$ be a subgraph of $\mathcal{G}_{\infty}$ with partite sets $X$ and $Y$ such that $m, n>2$. Then either $X \subset 6 \mathbb{N}_{0}$ and $Y \cap 6 \mathbb{N}_{0}=\emptyset$ or $Y \subset 6 \mathbb{N}_{0}$ and $X \cap 6 \mathbb{N}_{0}=\emptyset$.

Proof. If neither $X \not \subset 6 \mathbb{N}_{0}$ nor $X \cap 6 \mathbb{N}_{0} \neq \emptyset$, then either there exist $a \in X \cap 6 \mathbb{N}_{0}$ and $\{b, c\} \subset$ $X \cap\left(\mathbb{N}_{0} \backslash 6 \mathbb{N}_{0}\right)$ or there exist $\{b, c\} \subset X \cap 6 \mathbb{N}_{0}$ and $a \in X \cap\left(\mathbb{N}_{0} \backslash 6 \mathbb{N}_{0}\right)$.

If $a \in X \cap 6 \mathbb{N}_{0}$ and $\{b, c\} \subset X \cap\left(\mathbb{N}_{0} \backslash 6 \mathbb{N}_{0}\right)$, then $\left|Y \cap 6 \mathbb{N}_{0}\right| \leq 1$ by Lemma 4.9. Again for any $d \in Y \cap\left(\mathbb{N}_{0} \backslash 6 \mathbb{N}_{0}\right)$, Lemma 4.8 forces $b-6=d=c+6$ assuming $b>c$, without loss of generality. Hence $\left|Y \cap\left(\mathbb{N}_{0} \backslash 6 \mathbb{N}_{0}\right)\right| \leq 1$. Therefore, $|Y|=\left|Y \cap 6 \mathbb{N}_{0}\right|+\left|Y \cap\left(\mathbb{N}_{0} \backslash 6 \mathbb{N}_{0}\right)\right| \leq 2$, a contradiction.

If $\{b, c\} \subset X \cap 6 \mathbb{N}_{0}$ and $a \in X \cap\left(\mathbb{N}_{0} \backslash 6 \mathbb{N}_{0}\right)$, then $Y \cap 6 \mathbb{N}_{0}=\emptyset$ as otherwise each vertex of $Y \cap 6 \mathbb{N}_{0}$ must be adjacent to both $b$ and $c$ forcing them to be the same vertex by Lemma 4.9. On the other hand, if $d \in Y \cap\left(\mathbb{N}_{0} \backslash 6 \mathbb{N}_{0}\right)$, then $d=a-6$ or $d=a+6$ by Lemma 4.8. This implies $|Y|=\left|Y \cap 6 \mathbb{N}_{0}\right|+\left|Y \cap\left(\mathbb{N}_{0} \backslash 6 \mathbb{N}_{0}\right)\right| \leq 2$, a contradiction. So either $X \subset 6 \mathbb{N}_{0}$ or $X \cap 6 \mathbb{N}_{0}=\emptyset$. If $X \subset 6 \mathbb{N}_{0}$, then $Y \cap 6 \mathbb{N}_{0}=\emptyset$ by Lemma 4.9. If $X \cap 6 \mathbb{N}_{0}=\emptyset$, then $Y \cap\left(\mathbb{N}_{0} \backslash 6 \mathbb{N}_{0}\right)=\emptyset$ by Lemma 4.8. For if $x \in X$ is adjacent to $x-6, x+6 \in Y$, then $x+6$ can be adjacent to only $x$ and $x+12$. But $x+12$ is not adjacent to $x-6$.

In the next result we will also capture the case where at least one of the partite sets have exactly two vertices while the other one has at least four of them.

Theorem 4.11. Let $K_{2, n}$ be a subgraph of $\mathcal{G}_{\infty}$ with partite sets $X$ and $Y$ such that $|X|=2$ and $|Y|=n>3$. Then either $X \subset 6 \mathbb{N}_{0}$ and $Y \cap 6 \mathbb{N}_{0}=\emptyset$, or $X \cap 6 \mathbb{N}_{0}=\emptyset$ and $\left|Y \cap\left(\mathbb{N}_{0} \backslash 6 \mathbb{N}_{0}\right)\right| \leq 1$. 
Proof. Suppose $X=\{a, b\}$. Without loss of generality, let $a \in 6 \mathbb{N}_{0}$ and $b \in\left(\mathbb{N}_{0} \backslash 6 \mathbb{N}_{0}\right)$. Then $\left|Y \cap 6 \mathbb{N}_{0}\right| \leq 1$ and $\left|Y \cap\left(\mathbb{N}_{0} \backslash 6 \mathbb{N}_{0}\right)\right| \leq 2$ by Lemma 4.9 and 4.8. Therefore, $|Y| \leq 3$, a contradiction. Hence either $X \subset 6 \mathbb{N}_{0}$ or $X \cap 6 \mathbb{N}_{0}=\emptyset$. If $X \subset 6 \mathbb{N}_{0}$, then $Y \cap 6 \mathbb{N}_{0}=\emptyset$ by Lemma 4.9. If $X \cap 6 \mathbb{N}_{0}=\emptyset$, then $a$ and $b$ can have at most one common neighbour $c$ such that $c \in\left(\mathbb{N}_{0} \backslash 6 \mathbb{N}_{0}\right)$ by Lemma 4.8 .

Now let us try to understand the structure of independent sets in $\mathcal{G}_{\infty}$. Of course, as $\mathcal{G}_{\infty}$ is a bipartite graph, there are at least two distinct (and disjoint) independent sets in the form of the two partite sets. But how big can an independent set consisting of only consecutive (non-negative) even numbers be? We answer this question in the following result.

Theorem 4.12. There exist arbitrarily large independent sets containing consecutive (non-negative) even numbers in $\mathcal{G}_{\infty}$.

Proof. Given any $n \in \mathbb{N}$, the set $R=\{(2 n) !+2,(2 n) !+3, \ldots,(2 n) !+(2 n)\}$ is a set of consecutive composite numbers. Hence no two vertices in the set

$$
S=\{(2 n) !+2,(2 n) !+4, \ldots,(2 n) !+2 n\}
$$

are adjacent to each other as $\frac{a+b}{2} \in R$ for all $a, b \in S$. Hence $S$ is an independent set containing $n$ consecutive (non-negative) even numbers in $\mathcal{G}_{\infty}$.

\section{Conclusions}

We conclude the paper with an interesting observation that the graphs $\mathcal{G}_{\mathcal{E}_{n}^{*}}\left(\mathcal{P}_{1}\right)$ are Hamiltonian for all even $n$ with $4 \leqslant n \leqslant 58$, where $\mathcal{E}_{n}^{*}=\mathcal{E}_{n} \backslash\{0\}$ and $\mathcal{P}_{1}=\mathcal{P} \cup\{1\}$ (cf. Appendix A). Since the graph $\mathcal{G}_{\mathcal{E}_{n}^{*}}\left(\mathcal{P}_{1}\right)$ is bipartite, there cannot be any odd cycle in the graph. But it follows from the above observations that $\mathcal{G}_{\mathcal{E}_{n}^{*}}\left(\mathcal{P}_{1}\right)$ has a Hamiltonian path (i.e., a spanning path) for all odd $n$ with $5 \leqslant n \leqslant 57$ for if $\mathcal{G}_{\mathcal{E}_{2 m}^{*}}\left(\mathcal{P}_{1}\right)$ is Hamiltonian, then deleting the vertex corresponding to $2 m$ from any of its Hamiltonian cycle, we get a Hamiltonian path of $\mathcal{G}_{\mathcal{E}_{2 m-1}^{*}}\left(\mathcal{P}_{1}\right)$. Thus $\mathcal{G}_{\mathcal{E}_{n}^{*}}\left(\mathcal{P}_{1}\right)$ has a Hamiltonian path for all $n$ with $4 \leqslant n \leqslant 58$. The following is an interesting Hamiltonian path of $\mathcal{G}_{\mathcal{E}_{58}^{*}}\left(\mathcal{P}_{1}\right)$ that starts with 2 , ends at 116 and covers all even integers in between them:

$2,4,6,8,14,12,10,16,18,20,26,32,30,28,34,24,22,36,38,44,42,40,46,48,58,60,62,56,50,72,70$, $64,54,52,66,68,74,84,82,76,90,88,78,80,86,92,102,100,94,108,98,96,106,112,114,104,110,116$.

Let us call two even natural numbers conjugate to each other if $\{a, b\}=\{p-q, p+q\}$ for some $p, q$ each of which is either an odd prime or 1 . We see that there is a sequence of even natural numbers up to 1000 such that any two consecutive numbers in this sequence are conjugate to each other (cf. Appendix B). Now these observations lead to the following questions:

1. Does there exist a sequence of all even natural numbers such that any two consecutive numbers in this sequence are conjugate to each other? 
2. If the answer to the above question is negative, then what is the least value of $m$ such that $\mathcal{G}_{\mathcal{E}_{2 m}^{*}}\left(\mathcal{P}_{1}\right)$ is not Hamiltonian?

\section{Acknowledgements:}

We are grateful to the learned referees for their valuable suggestions and corrections which definitely improved and enhanced the paper. This research is partially supported by Jadavpur University under RUSA 2.0 Scheme (R-11/742/19 dated 27.06.2019) to the third author and partially by NSF under award CCF-1907738, which supported the second author in part.

\section{References}

[1] J. Bang-Jensen and G. Gutin, Classes of Directed Graphs. Springer Monographs in Mathematics. Springer, Cham. 2018.

[2] J. Bang-Jensen and F. Havet, Tournaments and Semicomplete Digraphs, In: Bang-Jensen J., Gutin G. (eds) Classes of Directed Graphs. Springer Monographs in Mathematics. Springer, Cham. 2018.

[3] A. Basu, S. Das, S. Ghosh and M. Sen, Circular-arc bigraphs and its subclasses, J. Graph Theory 73 (2013), $361-376$

[4] L. A. Berry, F. Havet, C. L. Sales, B. Reed, S. Thomasse, Oriented trees in digraphs, Discrete Math., 313 (2013), 967-974.

[5] J. R Chen, On the representation of a large even integer as the sum of a prime and the product of at most two primes, Kexue Tongbao, 11 (1966), 385-386.

[6] J. B. Jensen and G. Z. Gutin, Digraphs Theory, Algorithm and Applications, Springer, 2009.

[7] L. Kronecker, Vorlesungen über Zahlentheorie, I. p. 68, Teubner, Leipzig, 1901.

[8] E. Maillet, L'intermédiaire des math., 12 (1905), 108.

[9] Z. K. Min, Longest paths and cycles in bipartite oriented graphs, J. Graph Theory, 11 (1987), 339-348.

[10] J. Monsalve and J. Rada, Oriented bipartite graphs with minimal trace norm, Linear and Multilinear Algebra, 67 (2019), 1121-1131.

[11] J. Richstein, Verifying the goldbach conjecture up to $4 \times 10^{14}$, Mathematics of Computation, 70 (2000), 17451749.

[12] J. Riguet, Les relations des Ferrers, C. R. Acad. Sci. Paris, 232 (1951), 1729.

[13] J. Shen, On the girth of digraphs, Discrete Math., 211 (2000), 167-181.

[14] Terence Tao, Every odd number greater than 1 is the sum of at most five primes, Math. Comput., 83 (2014), 997-1038.

[15] I. M. Vinogradov, Representation of an odd number as a sum of three primes, Comptes Rendus (Doklady) de l'Academy des Sciences de l'USSR, 15 (1937), 191-294.

[16] H. Wang, Partition of a directed bipartite graph into two directed cycles, Discrete Math., 160 (1996), 283-289.

[17] D. B. West, Introduction to Graph Theory, Prentice-Hall of India Pvt.Ltd. 2003.

[18] A. Yeo, Semicomplete Multipartite Digraphs, In: Bang-Jensen J., Gutin G. (eds) Classes of Directed Graphs. Springer Monographs in Mathematics. Springer, Cham. 2018.

[19] Yitang Zhang, Bounded gaps between primes, Annals of Mathematics, 179 (2014), 1121-1174. 


\section{Appendix A}

\begin{tabular}{|c|c|}
\hline $\begin{array}{c}\text { Number of } \\
\text { Vertices }\end{array}$ & Hamiltonian Cycle \\
\hline 4 & $(4,2,8,6,4)$ \\
\hline 6 & $(4,6,8,2,12,10,4)$ \\
\hline 8 & $(4,2,8,14,12,10,16,6,4)$ \\
\hline 10 & $(4,2,8,6,16,10,12,14,20,18,4)$ \\
\hline 12 & $(4,2,8,6,16,10,12,22,24,14,20,18,4)$ \\
\hline 14 & $(4,2,8,6,28,18,16,22,12,26,20,14,24,10,4)$ \\
\hline 16 & $(4,2,8,6,16,10,12,22,24,14,20,26,32,30,28,18,4)$ \\
\hline 18 & $(4,2,8,6,16,10,12,14,20,18,28,34,24,22,36,26,32,30,4)$ \\
\hline 20 & $(4,2,8,6,16,10,12,22,36,38,24,14,20,26,32,30,28,34,40,18,4)$ \\
\hline 22 & $(4,2,8,6,16,10,12,14,20,18,28,34,40,42,32,26,36,22,24,38,44,30,4)$ \\
\hline 24 & $(4,2,8,6,16,10,12,14,20,18,28,30,32,26,36,22,24,34,40,46,48,38,44,42,4)$ \\
\hline 26 & $(4,2,8,6,16,10,12,14,20,18,28,34,40,46,36,22,24,50,44,38,48,26,32,30,52,42,4)$ \\
\hline 28 & $(4,2,8,6,16,10,12,14,20,18,28,30,56,50,44,38,24,22,36,46,40,34,48,26,32,54,52,42,4)$ \\
\hline 30 & $(4,2,8,6,16,10,12,14,20,18,28,30,56,50,44,38,24,22,36,46,40,34,48,58,60,26,32,54,52,42,4)$ \\
\hline 32 & $(4,2,8,6,16,10,12,14,20,18,28,30,32,26,36,22,24,34,40,46,48,38,44,50,56,62,60,58,64,54,52$, \\
\hline 34 & $\begin{array}{r}(4,2,8,6,16,10,12,14,20,18,28,30,32,26,36,22,24,34,40,46,48,38,44,50,56,62,60,58,64,54,68 \\
66,52,42,4)\end{array}$ \\
\hline 36 & $\begin{array}{r}(4,2,8,6,16,10,12,14,20,18,28,30,32,26,36,22,24,34,40,46,48,38,44,50,72,70,64,58,60,62,56, \\
66,68,54,52,42,4)\end{array}$ \\
\hline 38 & $\begin{array}{r}(4,2,8,6,16,10,12,14,20,18,28,30,32,26,36,22,24,34,40,46,48,38,44,50,56,62,60,58,64,54,52, \\
66,68,74,72,70,76,42,4)\end{array}$ \\
\hline 40 & $\begin{array}{r}(4,2,8,6,16,10,12,14,20,18,28,30,32,26,36,22,24,34,40,42,52,54,68,74,48,38,44,50,56,62,72 \\
46,60,58,64,70,76,66,80,78,4)\end{array}$ \\
\hline 42 & $\begin{array}{r}(4,2,8,6,16,10,12,14,20,18,28,30,32,26,36,22,24,34,40,42,44,38,48,46,60,58,64,70,76,82,84 \\
50,56,62,72,74,68,54,52,66,80,78,4)\end{array}$ \\
\hline 44 & $\begin{array}{r}(4,2,8,6,16,10,12,14,20,18,28,30,32,26,36,22,24,34,40,42,52,66,68,74,48,38,44,50,56,62,84 \\
58,64,70,76,82,60,46,72,86,80,54,88,78,4)\end{array}$ \\
\hline 46 & $\begin{array}{r}(4,2,8,6,16,10,12,14,20,18,28,30,32,26,36,22,24,34,40,42,44,38,48,46,60,58,64,70,76,82,84 \\
50,56,62,72,74,68,54,52,66,92,86,80,78,88,90,4)\end{array}$ \\
\hline 48 & $\begin{array}{r}(4,2,8,6,16,10,12,14,20,18,28,30,32,26,36,22,24,34,40,42,44,38,48,46,60,58,64,70,76,82,96 \\
50,56,62,72,94,84,74,68,54,52,66,92,86,80,78,88,90,4)\end{array}$ \\
\hline 50 & $\begin{array}{r}(4,2,8,6,16,10,12,14,20,18,28,30,32,26,36,22,24,34,40,46,48,38,44,42,100,94,72,62,56,50,96 \\
98,60,58,64,70,76,82,84,74,68,54,52,66,92,86,80,78,88,90,4)\end{array}$ \\
\hline 52 & $\begin{array}{r}(4,2,8,6,16,10,12,14,20,18,28,30,32,26,36,22,24,34,40,46,48,38,44,42,104,102,100,94,72,62,56 \\
50,96,98,60,58,64,70,76,82,84,74,68,54,52,66,92,86,80,78,88,90,4)\end{array}$ \\
\hline 54 & $\begin{array}{r}(4,2,8,6,16,10,12,14,20,18,28,30,32,26,36,22,24,34,40,46,48,38,44,42,104,102,100,106,108,94,72 \\
62,56,50,96,98,60,58,64,70,76,82,84,74,68,54,52,66,92,86,80,78,88,90,4)\end{array}$ \\
\hline 56 & $\begin{array}{r}(4,10,16,6,8,14,14,20,18,28,30,32,26,36,22,24,34,40,46,48,38,44,42,100,102,104,110,108,106,112 \\
90,88,78,80,86,92,66,52,54,68,74,84,82,76,70,64,58,60,98,96,50,56,62,72,94,12,2,4)\end{array}$ \\
\hline 58 & $\begin{array}{r}(6,4,2,8,14,12,10,16,18,20,26,32,30,28,34,24,22,36,38,44,42,40,46,48,58,60,62,56,50,72,70,64,54 \\
52,66,68,74,84,82,76,90,88,78,80,86,92,102,116,110,104,114,112,106,96,98,108,94,100,6)\end{array}$ \\
\hline
\end{tabular}




\section{Appendix B}

A Hamiltonian path in the graph $\mathcal{G}_{\mathcal{E}_{500}^{*}}\left(\mathcal{P}_{1}\right)$,

(A sequence of even natural numbers up to 1000 where

any pair of consecutive numbers are conjugate to each other).

$\{22,16,10,4,2,8,14,20,26,32,54,52,42,80,86,92,102,112,106,100,94,12,514,408$, 394, 400, 138, 284, 278, 288, 250, 204, 658, 664, 30, 136, 142, 120, 74, 68, 6, 340, 334, 328, 294, $152,146,528,586,372,362,356,350,344,18,28,34,40,46,36,430,436,442,576,662,656$, $650,644,638,24,338,636,710,672,682,516,502,496,798,844,642,776,770,324,218,228$, 926, 932, 390, 508, 330, 292, 210, 304, 310, 316, 222, 244, 238, 264, 482, 476, 282, 416, 422, 720 , $598,604,610,616,622,504,82,76,70,64,58,420,578,300,322,996,998,984,982,900,914$, $908,906,800,794,180,122,84,842,836,78,88,114,412,406,108,166,160,154,148,366,592$, 450, 764, 758, 60, 778, 768, 634, 628, 66, 812, 806, 72, 626, 468, 206, 540, 698, 704, 582, 940, 934, $588,674,680,686,552,646,640,762,752,630,808,814,708,746,740,738,700,594,872,882$, $892,870,788,786,172,834,688,694,948,986,980,974,968,750,268,274,280,286,252,374$, $492,466,460,454,312,614,620,618,676,670,972,970,964,942,716,722,696,818,824,830$, $828,946,960,242,236,230,224,702,920,954,248,254,260,266,272,606,308,314,320,306$, $388,414,332,666,472,726,728,654,632,246,712$, 706, 732, 554, 560, 566, 572, 966, 928, 714, $724,730,736,742,660,262,924,898,816,958,936,902,864,118,780,862,856,850,804,962$, $876,718,684,938,944,950,956,498,220,226,232$, 126, 352, 346, 48, 38, 44, 50, 56, 62, 96, 98 , 104, 110, 116, 198, 784, 790, 648, 734, 612, 190, 196, 202, 192, 890, 884, 878, 840, 886, 880, 874, $852,866,860,854,912,326,888,434,428,894,832,826,820,774,548,930,212,150,296,290$, $132,410,456,562,480,494,488,510,532,426,452,446,440,318,524,518,360,478,216,418$, 424, 342, 404, 398, 276, 470, 464, 458, 624, 298, 600, 766, 772, 270, 448, 186, 952, 486, 652, 474, $608,534,692,546,580,574,568,354,512,506,500,378,368,174,208,214,168,158,156,602$, $596,590,584,258,200,194,188,234,392,386,380,162,164,170,176,182,144,490,484,522$, 140, 134, 128, 90, 256, 462, 556, 558, 536, 918, 520, 526, 432, 550, 544, 538, 396, 922, 916, 438, $364,370,376,382$, 240, 302, 444, 530, 384, 542, 336, 178, 184, 402, 124, 130, 348, 358, 756, 838, $564,782,792,802,744,910,904,822,896,810,848,858,868,570,796,690,748,754,760,678$, $668,846,992,990,976,978,988,994,1000\}$ 